\title{
Well-posedness and nonsmooth Lyapunov pairs for state-dependent maximal monotone differential inclusions
}

\author{
Ba Khiet LE \\ Instituto de Ciencias de la Ingeniería, Universidad de O'Higgins, Rancagua, Chile \\ lebakhiet@gmail.com
}

March 24, 2022

\begin{abstract}
In this paper, we introduce for the first time a class of state-dependent maximal monotone differential inclusions. Then the existence and uniqueness of solutions are obtained by using an implicit discretization scheme and a kind of hypo-monotonicity assumption respectively. In addition, a characterization for nonsmooth Lyapunov pairs associated with such systems is provided. Our result can be applied to study state-dependent sweeping processes and Lur'e dynamical systems. It is new even the involved maximal monotone operators depend only on the time.
\end{abstract}

Keywords Differential inclusions, maximal monotone operators , state-dependent, Lur'e systems, sweeping processes

AMS subject classifications 49J40, 47J22, 49K40, 37B25

\section{Contents}

1 Introduction $\quad 2$

2 Mathematical backgrounds 3

3 Well-posedness of the problem 6

4 Stability analysis by using nonsmooth Lyapunov pairs

5 Applications for sweeping processes and Lur'e dynamical systems 20

5.1 State-dependent sweeping processes . . . . . . . . . . . . . . . 20

5.2 State-dependent Lur'e dynamical systems . . . . . . . . . . . . . . . . 21

6 Conclusion 


\section{Introduction}

The paper is dedicated to studying the well-posedness and Lyapunov stability for a new class of state-dependent maximal monotone differential inclusions under perturbations as follows

$$
\left\{\begin{array}{l}
\dot{x}(t) \in f(t, x(t))-A_{t, x(t)}(x(t)), \text { a.e. } t \geq 0, \\
x\left(t_{0}\right)=x_{0} \in \operatorname{dom}\left(A_{t_{0}, x_{0}}\right),
\end{array}\right.
$$

where $f:[0,+\infty) \times \mathbb{R}^{n} \rightarrow \mathbb{R}^{n}$ is a continuous function and $A_{t, x}: \mathbb{R}^{n} \rightarrow \mathbb{R}^{n}$ is a maximal monotone operator for each $(t, x) \in[0,+\infty) \times \mathbb{R}^{n}$, i.e., the monotone operator $A$ depends on both time and state. The classical maximal differential inclusions when $A$ is a fixed maximal monotone operator have been fruitfully studied in the literature, see for examples [5, 6]. There are also various works for the case of time-dependence, i.e., $A_{t, x} \equiv A_{t}$ (see [13, 15, 17, 26, 32] and the reference therein). Among important contributions are sweeping processes [3, 16, 18, 22, 23, 24, 25, 30, 31, Skorohod problem [29], hysteresis operators [14] and recently Lur'e dynamical systems [1, 7, 8, 9, 10, 19, 27, 28,. In particular, when $A_{t, x} \equiv N_{C(t)}$, the normal cone of a moving closed convex set, one obtains the sweeping processes

$$
\left\{\begin{array}{l}
\dot{x}(t) \in f(t, x(t))-N_{C(t)}(x(t)), \text { a.e. } t \geq 0, \\
x\left(t_{0}\right)=x_{0},
\end{array}\right.
$$

which were introduced and thoroughly studied by J. J. Moreau [22, 23, 24, 25] in the seventies. Sweeping processes can be used for granular material, quasi-static evolution in elastoplasticity, nonsmooth mechanics, convex optimization, modeling of crowd motion, mathematical economics, switched electrical circuits. Another interesting application is Lur'e dynamical systems, which is of great interest in engineering, control theory and applied mathematics [1, 7, 9, 10, 20, 28. The systems comprise an interconnection between a smooth ordinary differential equation with a possibly set-valued feedback.

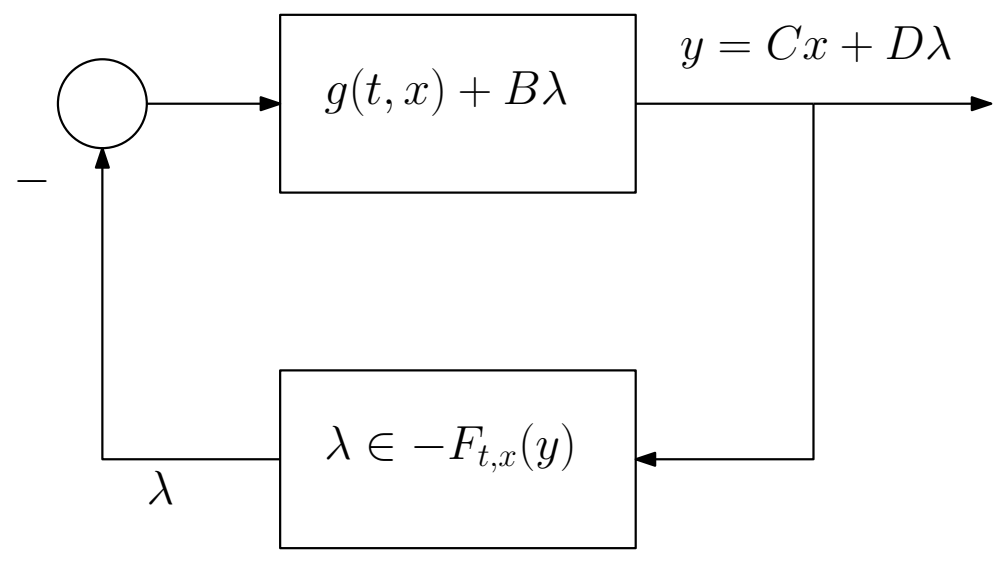

Figure 1: Lur'e dynamical systems with state-dependent set-valued feedback. 
In detail, let us consider the following systems

$$
\left\{\begin{array}{l}
\dot{x}(t)=g(t, x(t))+B \lambda(t) \text { a.e. } t \geq 0 \\
y(t)=C x(t)+D \lambda(t) \\
\lambda(t) \in-F_{t, x(t)}(y(t)), t \geq 0 \\
x(0)=x_{0}
\end{array}\right.
$$

where

- $g:[0, T] \times \mathbb{R}^{n} \rightarrow \mathbb{R}^{n}$ is a continuous function;

- $B: \mathbb{R}^{m} \rightarrow \mathbb{R}^{n}, C: \mathbb{R}^{n} \rightarrow \mathbb{R}^{m}, D: \mathbb{R}^{m} \rightarrow \mathbb{R}^{m}$ are some matrices;

- $F:[0, T] \times \mathbb{R}^{n} \times \mathbb{R}^{m} \rightrightarrows \mathbb{R}^{m}$ is a set-valued mapping such that for each $(t, x) \in[0, T] \times \mathbb{R}^{n}$, the operator $F_{t, x}(\cdot)$ is maximal monotone.

After some simple computations, we can reduce (3) into the following first order differential inclusions

$$
\left\{\begin{array}{l}
\dot{x}(t) \in g(t, x(t))-B\left(F_{t, y}^{-1}+D\right)^{-1} C x(t) \text { a.e. } t \geq 0, \\
x\left(t_{0}\right)=x_{0},
\end{array}\right.
$$

which is a particular form of (1). The case of stationary $F$ were considered in [1, 7, 8, 9, 10, while the time-dependent case was studied in [28] for $F_{t} \equiv N_{C(t)}$. The particular state-dependent case $F_{t, y} \equiv N_{C(t, y)}$ was considered in [19] recently.

To the best of our knowledge, there is still no research for general state-dependent maximal monotone differential inclusions, which is our motivation to fill this gap. On the other hand, Lyapunov stability of solutions through Lyapunov functions is always a nice property for any systems that people want to acquire. Smooth Lyapunov functions may limit applications due to the intrinsic nonsmoothness of many problems. Therefore, it is natural for us to consider nonsmooth Lyapunov pairs by using proximal analysis (see, e.g., [3, 4, 8, 9, 12, and references therein for smooth as well as nonsmooth Lyapunov functions for the stationary case and time-dependent sweeping processes). Our result is new even the involved maximal monotone operator depends only on the time.

The paper is organized as follows. In Section 2, we recall some useful definitions and results which will be used later. Then the well-posedness of problem (1) is considered in Section 3 by using an implicit discretization scheme and a kind of hypo-monotonicity assumption. In Section 4, we provide a charactization for lower semi-continous Lyapunov pairs for (1) with some illustrative examples. The obtained results can be applied to study the state-dependent sweeping processes and Lur'e dynamical systems in Section 5. Finally, some conclusions end the paper in Section 6.

\section{Mathematical backgrounds}

Let us first introduce some notation that will be used in the sequel. Denote by $\langle\cdot, \cdot\rangle,\|\cdot\|, \mathbb{B}$ the scalar product, the corresponding norm and the closed unit ball in Euclidean spaces. Let be given 
a closed, convex set $K \subset \mathbb{R}^{n}$. The distance and the projection from a point $s$ to $K$ are defined respectively by

$$
d(s, K):=\inf _{x \in K}\|s-x\|, \operatorname{proj}(s, K):=x \in K \text { such that } d(s, K)=\|s-x\| .
$$

The minimal norm element of $K$ is defined by

$$
K^{0}:=\operatorname{proj}(0 ; K)
$$

The normal cone of $K$ is given by

$$
N(K, x)=\left\{x^{*}:\left\langle x^{*}, y-x\right\rangle \leq 0, \quad \forall y \in K\right\} .
$$

The Hausdorff distance between two closed, convex sets $K_{1}, K_{2}$ is given by

$$
d_{H}\left(K_{1}, K_{2}\right):=\max \left\{\sup _{x_{1} \in K_{1}} d\left(x_{1}, K_{2}\right), \sup _{x_{2} \in K_{2}} d\left(x_{2}, K_{1}\right)\right\}
$$

Now let $K$ be a closed subset of $\mathbb{R}^{n}$. The proximal normal cone, the limiting normal cone and the Clarke normal cone of $K$ at $x$ are defined [11, 21] respectively as

$$
\begin{gathered}
N^{P}(K, x):=\left\{\xi \in \mathbb{R}^{n}: \exists \delta>0 \text { s.t. }\langle\xi, y-x\rangle \leq \delta\|y-x\|^{2} \text { for all } y \in K\right\}, \\
N^{L}(K, x):=\left\{\xi \in \mathbb{R}^{n}: \exists \xi_{n} \rightarrow \xi \text { and } \xi_{n} \in N^{P}\left(K, x_{n}\right), x_{n} \rightarrow x \text { in } K\right\},
\end{gathered}
$$

and

$$
N^{C}(K, x):=\overline{\operatorname{co}} N^{L}(K, x) .
$$

It is easy to see that if $K$ is also convex then $N^{P}(K, x)=N^{L}(K, x)=N^{C}(K, x)=N(K, x)$.

Definition 2.1 Let $\varphi: \mathbb{R}^{n} \rightarrow \mathbb{R} \cup\{+\infty\}$ be a proper lower semi-continuous function and $x \in \mathbb{R}^{n}$ at which $\varphi$ is finite. The proximal subdifferential, singular subdifferential and Clarke subdifferential of $\varphi$ at $x$ are defined respectively by

$$
\begin{gathered}
\partial^{P} \varphi(x):=\left\{\xi \in \mathbb{R}^{n}:(\xi,-1) \in N_{\mathrm{epi} \varphi}^{P}(x, \varphi(x))\right\}, \\
\partial^{\infty} \varphi(x):=\left\{\xi \in \mathbb{R}^{n}:(\xi, 0) \in N_{\text {epi } \varphi}^{L}(x, \varphi(x))\right\},
\end{gathered}
$$

and

$$
\partial^{C} \varphi(x):=\left\{\xi \in \mathbb{R}^{n}:(\xi,-1) \in N_{\text {epi } \varphi}^{C}(x, \varphi(x))\right\} .
$$

It is known that $\xi \in \partial^{\infty} \varphi(x)$ if and only if there exist sequences $\left(\alpha_{k}\right)_{k \in \mathbb{N}} \subset \mathbb{R}_{+},\left(x_{k}\right)_{k \in \mathbb{N}},\left(\xi_{k}\right)_{k \in \mathbb{N}}$ such that $\alpha_{k} \rightarrow 0^{+}, x_{k} \rightarrow_{\varphi} x, \xi_{k} \in \partial^{P} \varphi\left(x_{k}\right)$ and $\alpha_{k} \xi_{k} \rightarrow \xi$ (see, e.g., [21]).

Definition 2.2 A matrix $P \in \mathbb{R}^{n \times n}$ is called

- positive semidefinite if for all $x \in \mathbb{R}^{n}$, we have

$$
\langle P x, x\rangle \geq 0
$$

- positive definite if there exists $\alpha>0$ such that for all $x \in \mathbb{R}^{n}$, we have

$$
\langle P x, x\rangle \geq \alpha\|x\|^{2} .
$$


We have the following fundamental lemma.

Lemma 2.1 Let $D$ be a positive semidefinite matrix. Then there exists some constant $c_{1}>0$ such that for all $x \in \operatorname{rge}\left(D+D^{T}\right)$, we have:

$$
\langle D x, x\rangle \geq c_{1}\|x\|^{2} .
$$

Proof. If $D$ is non-zero then $c_{1}$ can be chosen as the smallest positive eigenvalue of $D+D^{T}$.

Definition 2.3 A set-valued mapping $F: \mathbb{R}^{n} \rightrightarrows \mathbb{R}^{n}$ is called monotone if for all $x, y \in \mathbb{R}^{n}, x^{*} \in$ $F(x), y^{*} \in F(y)$, one has $\left\langle x^{*}-y^{*}, x-y\right\rangle \geq 0$. In addition, it is called maximal monotone if there is no monotone operator $G$ such that the graph of $F$ is contained strictly in the graph of $G$, i.e., if

$$
\left\langle x^{*}-y^{*}, x-y\right\rangle \geq 0,
$$

for all $\left(y, y^{*}\right)$ in the graph of $F$, then $x^{*} \in F(x)$.

Proposition 2.1 ([5, 6]) Let $H$ be a Hilbert space, $F: H \rightrightarrows H$ be a maximal monotone operator and let $\lambda>0$. Then

1) the resolvent of $F$ defined by $J_{F}^{\lambda}:=(I+\lambda F)^{-1}$ is a non-expansive and single-valued map from $H$ to $H$.

2) the Yosida approximation of $F$ defined by $F^{\lambda}:=\frac{1}{\lambda}\left(I-J_{F}^{\lambda}\right)=\left(\lambda I+F^{-1}\right)^{-1}$ satisfies

i) for all $x \in H, F^{\lambda}(x) \in F\left(J_{F}^{\lambda} x\right)$,

ii) $F_{\lambda}$ is Lipschitz continuous with constant $\frac{1}{\lambda}$ and also maximal monotone.

iii) If $x \in \operatorname{dom}(F)$, then $\left\|F^{\lambda} x\right\| \leq\left\|F^{0} x\right\|$, where $F^{0} x$ is the element of $F x$ of minimal norm.

Let us recall Minty's Theorem in the setting of Hilbert spaces (see [5, 6]).

Proposition 2.2 Let $H$ be a Hilbert space. Let $F: H \rightrightarrows H$ be a monotone operator. Then $F$ is maximal monotone if and only if $\operatorname{rge}(F+I)=H$.

Let be given two maximal monotone operators $F_{1}$ and $F_{2}$, we recall the definition of pseudo-distance between $F_{1}$ and $F_{2}$ introduced by Vladimirov [32] as follows

$$
\operatorname{dis}\left(F_{1}, F_{2}\right):=\sup \left\{\frac{\left\langle\eta_{1}-\eta_{2}, z_{2}-z_{1}\right\rangle}{1+\left|\eta_{1}\right|+\left|\eta_{2}\right|}: \eta_{i} \in F\left(z_{i}\right), z_{i} \in \operatorname{dom}\left(F_{i}\right), i=1,2\right\} .
$$

Lemma 2.2 [32] If $F_{i}=N_{A_{i}}$ where $A_{i}$ is a closed convex set $(i=1,2)$ then

$$
\operatorname{dis}\left(F_{1}, F_{2}\right)=d_{H}\left(A_{1}, A_{2}\right) .
$$

Lemma 2.3 [16] Let $F_{1}, F_{2}$ be two maximal monotone operators. For $\lambda>0, \delta>0$ and $x \in$ $\operatorname{dom}\left(F_{1}\right)$, we have

$$
\begin{aligned}
\left\|x-J_{F_{2}}^{\lambda}(x)\right\| & \leq \lambda\left\|F_{1}^{0} x\right\|+\operatorname{dis}\left(F_{1}, F_{2}\right)+\sqrt{\lambda\left(1+\left\|F_{1}^{0} x\right\|\right) \operatorname{dis}\left(F_{1}, F_{2}\right)} \\
& \leq \lambda\left\|F_{1}^{0} x\right\|+\operatorname{dis}\left(F_{1}, F_{2}\right)+\left(\delta \operatorname{dis}\left(F_{1}, F_{2}\right)+\frac{\lambda\left(1+\left\|F_{1}^{0} x\right\|\right)}{4 \delta}\right) \\
& \left.\leq \frac{\lambda\left(1+(4 \delta+1)\left\|F_{1}^{0} x\right\|\right)}{4 \delta}\right)+(1+\delta) \operatorname{dis}\left(F_{1}, F_{2}\right) .
\end{aligned}
$$


Lemma 2.4 [16] Let $F_{n}$ be a sequence of maximal monotone operators in a Hilbert space $H$ such that $\operatorname{dis}\left(F_{n}, F\right) \rightarrow 0$ as $n \rightarrow+\infty$ for some maximal monotone operator $F$. Suppose that $x_{n} \in$ $\operatorname{dom}\left(F_{n}\right)$ with $x_{n} \rightarrow x$ and that $y_{n} \in F_{n}\left(x_{n}\right)$ with $y_{n} \rightarrow y$ weakly for some $x, y \in H$. Then $x \in \operatorname{dom}(F)$ and $y \in F(x)$.

Let us end-up this section by recalling some versions of Gronwall's inequality.

Lemma 2.5 Let $\alpha>0$ and $\left(u_{n}\right),\left(\beta_{n}\right)$ be non-negative sequences satisfying

$$
u_{n} \leq \alpha+\sum_{k=0}^{n-1} \beta_{k} u_{k} \forall n=0,1,2, \ldots \quad\left(\text { with } \beta_{-1}:=0\right) .
$$

Then, for all $n$, we have

$$
u_{n} \leq \alpha \exp \left(\sum_{k=0}^{n-1} \beta_{k}\right) .
$$

Lemma 2.6 Let $T>0$ be given and $a(\cdot), b(\cdot) \in L^{1}([0, T] ; \mathbb{R})$ with $b(t) \geq 0$ for almost all $t \in[0, T]$. Let an absolutely continuous function $w:[0, T] \rightarrow \mathbb{R}_{+}$satisfy

$$
(1-\alpha) w^{\prime}(t) \leq a(t) w(t)+b(t) w^{\alpha}(t), \text { a.e. } t \in[0, T]
$$

where $0 \leq \alpha<1$. Then for all $t \in[0, T]$, we have

$$
w^{1-\alpha}(t) \leq w^{1-\alpha}(0) \exp \left(\int_{0}^{t} a(\tau) d \tau\right)+\int_{0}^{t} \exp \left(\int_{s}^{t} a(\tau) d \tau\right) b(s) d s .
$$

\section{Well-posedness of the problem}

In this section, the existence and uniqueness of solutions for problem (1) are studied by using an implicit approximation scheme inspired by [19] and a kind of hypo-monotonicity assumption repsectively. Let be given arbitrary $T>0$. First, we propose followings assumptions.

Assumption 1 For every $t \in[0, T]$ and $x \in \mathbb{R}^{n}$, the operator $A_{t, x}: \mathbb{R}^{n} \rightrightarrows \mathbb{R}^{n}$ be a maximal monotone operator such that

(1.1) there exists non-negative constants $L_{1}, L_{2}$ with $L_{2}<1$ such that

$$
\operatorname{dis}\left(A_{t, x}, A_{s, y}\right) \leq L_{1}|t-s|+L_{2}\|x-y\|, \quad \forall t, s \in[0, T] .
$$

(1.2) there exists $c_{A}>0$ such that

$$
\left\|A_{t, x}^{0}(y)\right\| \leq c_{A}(1+\|x\|+\|y\|), t \in[0, T], x, y \in \mathbb{R}^{n} .
$$

Assumption 2 Let $f:[0, T] \times \mathbb{R}^{n} \rightarrow \mathbb{R}^{n}$ be a continuous function. In addition, suppose that there exists $c_{f}>0$ such that

$$
\|f(t, x)\| \leq c_{f}(1+\|x\|), \forall t \in[0, T], x \in \mathbb{R}^{n} .
$$

We define the admissible set for (1) as follows:

$$
\mathcal{A}_{1}:=\left\{\left(t_{0}, x_{0}\right): x_{0} \in \operatorname{dom}\left(A_{t_{0}, x_{0}}\right)\right\} .
$$


Theorem 3.1 (Existence) Let Assumptions 1, 2 hold. Then for all $\left(t_{0}, x_{0}\right) \in \mathcal{A}_{1}$ with $0 \leq t_{0} \leq T$, the problem

$$
\dot{x}(t) \in-A_{t, x(t)} x(t)+f(t, x(t)) \text {, a.e. } t \in\left[t_{0}, T\right], x\left(t_{0}\right)=x_{0},
$$

has a solution $x(\cdot)$. In addition, we have

$$
\|\dot{x}(t)\| \leq m\left(x_{0}\right), \text { a.e. } t \in\left[t_{0}, t_{0}+1\right],
$$

where $m\left(x_{0}\right)>0$ is defined in (32) depending only on $x_{0}, L_{1}, L_{2}, c_{A}, c_{f}$ and $m(\cdot)$ is a continuous function w.r.t $x_{0}$. Therefore

$$
\|x(t)\| \leq\left\|x_{0}\right\|+m\left(x_{0}\right), \quad \forall t \in\left[t_{0}, t_{0}+1\right],
$$

Proof. We use an implicit scheme to approximate problem (1). In details, let $T^{\prime}=T-t_{0}$ and for each given positive integer $n$, we set $h_{n}=T^{\prime} / n$ and $t_{i}^{n}=t_{0}+i h$ for $0 \leq i \leq n$. We can construct the sequence $\left(x_{i}^{n}\right)_{0 \leq i \leq n}$ with $x_{0}^{n}=x_{0}$ as follows:

$$
\left\{\begin{array}{l}
y_{i}^{n}=x_{i}^{n}+h_{n} f\left(t_{i}^{n}, x_{i}^{n}\right), \\
x_{i+1}^{n} \in y_{i}^{n}-h_{n} A_{t_{i+1}^{n}, x_{i}^{n}}\left(x_{i+1}^{n}\right) .
\end{array}\right.
$$

Indeed, we can compute $x_{i+1}^{n}$ by

$$
x_{i+1}^{n}=\left(I+h_{n} A_{t_{i+1}^{n}, x_{i}^{n}}\right)^{-1}\left(y_{i}^{n}\right)=J_{A_{t_{i+1}^{n}, x_{i}^{n}}^{h_{n}}}\left(y_{i}^{n}\right) .
$$

Thus we obtain the following algorithm which is well-defined.

\section{Algorithm}

Initialization. Let $x_{0}^{n}:=x_{0}, y_{0}^{n}:=x_{0}^{n}+h_{n} f\left(t_{0}^{n}, x_{0}^{n}\right)$.

Iteration. For the current points $x_{i}^{n}$, we compute

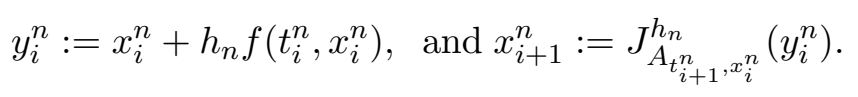

From (16), we have

$$
\begin{aligned}
& \left\|x_{i+1}^{n}-x_{i}^{n}\right\|=\left\|J_{A_{t_{i+1}^{n}, x_{i}^{n}}^{h_{n}}}\left(y_{i}^{n}\right)-x_{i}^{n}\right\|
\end{aligned}
$$

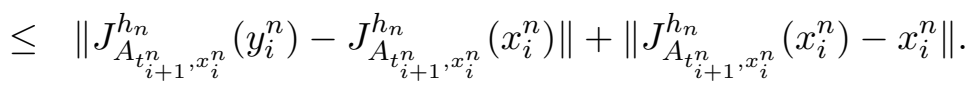

Since $J_{A_{t_{i+1}^{n}, x_{i}^{n}}^{n}}^{h_{n}}$ is non-expansive, one has

$$
\begin{aligned}
& \left\|J_{A_{t_{i+1}^{n}, x_{i}^{n}}^{h_{n}}}\left(y_{i}^{n}\right)-J_{A_{t_{i+1}^{n}, x_{i}^{n}}^{h_{n}}}\left(x_{i}^{n}\right)\right\| \leq\left\|y_{i}^{n}-x_{i}^{n}\right\| \\
& \leq h_{n}\left\|f\left(t_{i}^{n}, x_{i}^{n}\right)\right\| \leq h_{n} c_{f}\left(1+\left\|x_{i}^{n}\right\|\right) .
\end{aligned}
$$

Since $L_{2}<1$, we can always choose some constant $\delta>0$ such that

$$
\tilde{L}_{2}:=(1+\delta) L_{2}<1
$$


Note that $x_{i}^{n} \in \operatorname{dom}\left(A_{t_{i}^{n}, x_{i-1}^{n}}\right)$ for $i=0, . ., n$ with $x_{-1}^{n}:=x_{0}^{n}$, by using Lemma 2.3 and Assumption 1.1, we have

$$
\begin{aligned}
& \| J_{A_{t_{i+1}^{n}, x_{i}^{n}}^{h_{n}}\left(x_{i}^{n}\right)-x_{i}^{n} \|} \leq h_{n} \frac{1+(4 \delta+1)\left\|A_{t_{i}^{n}, x_{i-1}^{n}}^{0}\left(x_{i}^{n}\right)\right\|}{4 \delta}+(1+\delta) \operatorname{dis}\left(A_{t_{i+1}^{n}, x_{i}^{n}}, A_{t_{i}^{n}, x_{i-1}^{n}}\right) \\
& \leq h_{n} \frac{1+(4 \delta+1) c_{A}\left(1+\left\|x_{i}^{n}\right\|+\left\|x_{i-1}^{n}\right\|\right)}{4 \delta} \\
&\left.+(1+\delta) L_{1} h_{n}+(1+\delta) L_{2}\left\|x_{i}^{n}-x_{i-1}^{n}\right\|\right) .
\end{aligned}
$$

From (17), (18), 19) and (20), one has

$$
\begin{aligned}
\left\|x_{i+1}^{n}-x_{i}^{n}\right\| & \left.\leq h_{n} c_{1}\left(1+\left\|x_{i+1}^{n}\right\|+\left\|x_{i}^{n}\right\|+\left\|x_{i-1}^{n}\right\|\right)\right) \\
& \left.+\tilde{L}_{2}\left\|x_{i}^{n}-x_{i-1}^{n}\right\|\right)
\end{aligned}
$$

where $\tilde{L}_{2}<1$ and

$$
c_{1}:=c_{f}+\frac{1+(4 \delta+1) c_{A}}{4 \delta}+(1+\delta) L_{1}
$$

Since $x_{-1}^{n}:=x_{0}^{n}$, we obtain

$$
\begin{aligned}
\left\|x_{i+1}^{n}-x_{i}^{n}\right\| & \leq h_{n} c_{1} \sum_{j=0}^{i} \tilde{L}_{2}^{j}\left(1+\left\|x_{i-j+1}^{n}\right\|+\left\|x_{i-j}^{n}\right\|+\left\|x_{i-j-1}^{n}\right\|\right) \\
& \leq h_{n} c_{1}\left(\frac{1}{1-\tilde{L}_{2}}+\sum_{j=0}^{i} \tilde{L}_{2}^{j}\left(\left\|x_{i-j+1}^{n}\right\|+\left\|x_{i-j}^{n}\right\|+\left\|x_{i-j-1}^{n}\right\|\right) .\right.
\end{aligned}
$$

Thus

$$
\begin{aligned}
\left\|x_{i+1}^{n}-x_{0}^{n}\right\| & \leq \sum_{j=0}^{i}\left\|x_{j+1}^{n}-x_{j}^{n}\right\| \\
& \leq h_{n} c_{1}\left(\frac{i+1}{1-\tilde{L}_{2}}+\left\|x_{i+1}^{n}\right\|+3 \sum_{j=0}^{i} \tilde{L}_{2}^{j} \sum_{j=0}^{i}\left\|x_{j}^{n}\right\|\right) \\
& \leq \frac{c_{1} T^{\prime}}{1-\tilde{L}_{2}}+h_{n} c_{1}\left\|x_{i+1}^{n}\right\|+\frac{3 h_{n} c_{1}}{1-\tilde{L}_{2}} \sum_{j=0}^{i}\left\|x_{j}^{n}\right\| .
\end{aligned}
$$

We can choose $n$ large enough such that $h_{n} c_{1}<1 / 2$. Then we have

$$
\left\|x_{i+1}^{n}\right\| \leq c_{2}+c_{3} h_{n} \sum_{j=0}^{i}\left\|x_{j}^{n}\right\|,
$$

where

$$
c_{2}:=2\left\|x_{0}\right\|+\frac{2 c_{1} T^{\prime}}{1-\tilde{L}_{2}}, \quad c_{3}:=\frac{6 c_{1}}{1-\tilde{L}_{2}} .
$$

Thus one has

$$
\left\|x_{i+1}^{n}\right\| \leq M_{1}:=c_{2} e^{c_{3} T^{\prime}}, i=0,1, \ldots, n-1,
$$


by using the discrete Gronwall's inequality in Lemma 2.5. From (22), we deduce that

$$
\left\|\frac{x_{i+1}^{n}-x_{i}^{n}}{h_{n}}\right\| \leq \frac{c_{1}\left(1+3 M_{1}\right)}{1-\tilde{L}_{2}}:=M_{2} .
$$

Now let us construct the sequences of functions $\left(x_{n}(\cdot)\right)_{n},\left(\theta_{n}(\cdot)\right)_{n},\left(\eta_{n}(\cdot)\right)_{n}$, on $\left[t_{0}, T\right]$ as follows: for $0 \leq i \leq n-1$, on $\left[t_{i}^{n}, t_{i+1}^{n}\right)$, we define

$$
x_{n}(t):=x_{i}^{n}+\frac{x_{i+1}^{n}-x_{i}^{n}}{h_{n}}\left(t-t_{i}^{n}\right)
$$

and

$$
\theta_{n}(t):=t_{i}^{n}, \quad \eta_{n}(t):=t_{i+1}^{n} .
$$

Thanks to 24$)$, for all $t \in\left(t_{i}^{n}, t_{i+1}^{n}\right)$, we have

$$
\left\|\dot{x}_{n}(t)\right\|=\left\|\frac{x_{i+1}^{n}-x_{i}^{n}}{h_{n}}\right\| \leq M_{2}
$$

and

$$
\sup _{t \in\left[t_{0}, T\right]}\left\{\left|\theta_{n}(t)-t\right|,\left|\eta_{n}(t)-t\right|\right\} \leq h_{n} \rightarrow 0 \text { as } n \rightarrow+\infty .
$$

Therefore $\left(x_{n}(\cdot)\right)_{n}$ is uniformly bounded and equi-Lipschitz continuous. As a consequence of ArzelàAscoli theorem, one can find a Lipschitz continuous function $x(\cdot):\left[t_{0}, T\right] \rightarrow \mathbb{R}^{n}$ and a subsequence, still denoted by $\left(x_{n}(\cdot)\right)_{n}$, satisfying:

- $x_{n}(\cdot)$ converges strongly to $x(\cdot)$ in $\mathcal{C}\left(\left[t_{0}, T\right] ; \mathbb{R}^{n}\right)$;

- $\dot{x}_{n}(\cdot)$ converges weakly to $\dot{x}(\cdot)$ in $L^{2}\left(\left[t_{0}, T\right] ; \mathbb{R}^{n}\right)$.

Particularly, we have $x(0)=x_{0}$. From (15), 25) and (26), we obtain

$$
\dot{x}_{n}(t) \in f\left(\theta_{n}(t), x_{n}\left(\theta_{n}(t)\right)\right)-A_{\eta_{n}(t), x_{n}\left(\theta_{n}(t)\right.}\left(x_{n}\left(\eta_{n}(t)\right)\right) .
$$

For each positive integer $n$, let us define the operators $\mathcal{A}, \mathcal{A}_{n}: L^{2}\left(\left[t_{0}, T\right] ; \mathbb{R}^{n}\right) \rightarrow L^{2}\left(\left[t_{0}, T\right] ; \mathbb{R}^{n}\right)$ as follows

$$
z^{*} \in \mathcal{A}(z) \Leftrightarrow z^{*}(t) \in A_{t, x(t)}(z(t)) \text { a.e. } t \in\left[t_{0}, T\right]
$$

and

$$
z^{*} \in \mathcal{A}_{n}(z) \Leftrightarrow z^{*}(t) \in A_{\eta_{n}(t), x_{n}\left(\theta_{n}(t)\right)}(z(t)) \text { a.e. } t \in\left[t_{0}, T\right] .
$$

Using Minty's theorem, it is easy to see that $\mathcal{A}_{n}, \mathcal{A}$ are maximal monotone operators since $A_{t, x(t)}$ 
and $A_{\eta_{n}(t), x_{n}\left(\theta_{n}(t)\right)}$ are maximal monotone for each $t \in\left[t_{0}, T\right]$. Furthermore, we have

$$
\begin{aligned}
& \operatorname{dis}\left(\mathcal{A}_{n}, \mathcal{A}\right) \\
= & \sup \left\{\frac{\int_{t_{0}}^{T}\left\langle z_{n}^{*}(t)-z^{*}(t), z_{n}(t)-z(t)\right\rangle d t}{1+\left\|z_{n}^{*}\right\|_{L^{2}}+\left\|z^{*}\right\|_{L^{2}}}: z_{n}^{*} \in \mathcal{A}_{n}\left(z_{n}\right), z^{*} \in \mathcal{A}(z)\right\} \\
\leq & \sup \left\{\frac{\int_{t_{0}}^{T} \operatorname{dis}\left(A_{\eta_{n}(t), x_{n}\left(\theta_{n}(t)\right)}, A_{t, x(t)}\right)\left(1+\left\|z_{n}^{*}(t)\right\|+\left\|z^{*}(t)\right\|\right) d t}{1+\left\|z_{n}^{*}\right\|_{L^{2}}+\left\|z^{*}\right\|_{L^{2}}}: z_{n}^{*} \in \mathcal{A}_{n}\left(z_{n}\right), z^{*} \in \mathcal{A}(z)\right\} \\
& \left(\operatorname{using} \text { the definition of } \operatorname{dis}\left(A_{\eta_{n}(t), x_{n}\left(\theta_{n}(t)\right)}, A_{t, x(t)}\right)\right) \\
\leq & \sup \left\{\frac{\int_{t_{0}}^{T}\left(L_{1}\left|\eta_{n}(t)-t\right|+L_{2} \| x_{n}\left(\theta_{n}(t)-x(t) \|\right)\left(1+\left\|z_{n}^{*}(t)\right\|+\left\|z^{*}(t)\right\|\right) d t\right.}{1+\left\|z_{n}^{*}\right\|_{L^{2}}+\left\|z^{*}\right\|_{L^{2}}}:\right. \\
& \left.z_{n}^{*} \in \mathcal{A}_{n}\left(z_{n}\right), z^{*} \in \mathcal{A}(z)\right\} \\
& (\operatorname{using} \operatorname{Assumption} 1) \\
\leq & \left(L_{1}\left\|\eta_{n}-I\right\|_{L^{2}}+L_{2}\left\|x_{n} \circ \theta_{n}-x\right\|_{L^{2}}\right) \sup \left\{\frac{1+\left\|z_{n}^{*}\right\|_{L^{2}}+\left\|z^{*}\right\|_{L^{2}}}{1+\left\|z_{n}^{*}\right\|_{L^{2}}+\left\|z^{*}\right\|_{L^{2}}}:\right. \\
& \left.z_{n}^{*} \in \mathcal{A}_{n}\left(z_{n}\right), z^{*} \in \mathcal{A}(z)\right\} \\
= & L_{1}\left\|\eta_{n}-I\right\|_{L^{2}}+L_{2}\left\|x_{n} \circ \theta_{n}-x\right\|_{L^{2}} \rightarrow 0,
\end{aligned}
$$

as $n \rightarrow+\infty$.

Note that $\dot{x}_{n}$ converges weakly to $\dot{x}$ in $L^{2}\left(\left[t_{0}, T\right] ; \mathbb{R}^{n}\right)$. Thus Assumption 2 allows us to deduce that

$$
\dot{x}_{n}-f\left(\theta_{n}(\cdot), x_{n} \circ \theta_{n}(\cdot)\right) \rightarrow \dot{x}-f(\cdot, x)
$$

weakly in $L^{2}\left(\left[t_{0}, T\right] ; \mathbb{R}^{n}\right)$. In addition, $x_{n} \circ \eta_{n}$ converges strongly $x$ in $L^{2}\left(\left[t_{0}, T\right] ; \mathbb{R}^{n}\right)$. Using Lemma 2.4 and (28), we have

$$
\dot{x}-f(\cdot, x) \in-\mathcal{A}(x)
$$

or equivalently

$$
\dot{x}(t)-f(t, x(t)) \in-A_{t, x(t)}(x(t)), \text { a.e. } t \in\left[t_{0}, T\right],
$$

which shows that $x(\cdot)$ is a solution of (1). Note that in 24) if we replace $T^{\prime}=T-t_{0}$ by 1 , we can obtain that

$$
\|\dot{x}(t)\| \leq m\left(x_{0}\right) \text {, a.e. } t \in\left[t_{0}, t_{0}+1\right],
$$

where

$$
\begin{aligned}
m\left(x_{0}\right) & :=c_{2} e^{c_{3}}=\left(2\left\|x_{0}\right\|+\frac{2 c_{1}}{1-\tilde{L}_{2}}\right) \exp \left(\frac{6 c_{1}}{1-\tilde{L}_{2}}\right) \\
& =\left(2\left\|x_{0}\right\|+\frac{2 c_{1}}{1-(1+\delta) L_{2}}\right) \exp \left(\frac{6 c_{1}}{1-(1+\delta) L_{2}}\right)
\end{aligned}
$$

and

$$
c_{1}:=c_{f}+\frac{1+(4 \delta+1) c_{A}}{4 \delta}+(1+\delta) L_{1}
$$

The proof is completed. 
Remark 3.1 (i) The result in Theorem 3.1 is still valid for infinite dimensional Hilbert spaces with some additional compactness assumption. Let us note that even the existence of solutions in infinite dimensional spaces for state-dependent sweeping processes, a particular case of (1), without any compactness assumption is still an open question.

(ii) The single-valued perturbation $f$ can be replaced standardly by a set-valued upper semi-continuous mapping with convex weakly compact values satisfying some linear growth condition.

Theorem 3.2 (Uniqueness) Let all assumptions in Theorem 3.1 hold. In addition, suppose that $f$ is Lipschitz continuous on bounded sets w.r.t the second variable and $A$ is hypo-monotone on bounded sets, in the sense that, for given $M>0$ there exist $k_{M}, l_{M}>0$ such that for all $t \in$ $[0, T], x_{i} \in \mathbb{R}^{n} \cap M \mathbb{B}, x_{i}^{*} \in A_{t, x_{i}}\left(x_{i}\right), i=1,2$ we have

$$
\left\langle x_{1}^{*}-x_{2}^{*}, x_{1}-x_{2}\right\rangle \geq-k_{M}\left\|x_{1}-x_{2}\right\|^{2},
$$

and

$$
\left\|f\left(t, x_{1}\right)-f\left(t, x_{2}\right)\right\| \leq l_{M}\left\|x_{1}-x_{2}\right\| .
$$

Then for each $\left(t_{0}, x_{0}\right) \in \mathcal{A}_{1}$, problem (1) has a unique solution on $\left[t_{0}, T\right]$.

Proof. Let $x_{1}(\cdot), x_{2}(\cdot)$ be two solutions of (1) with the same initial conditions $x_{1}\left(t_{0}\right)=x_{2}\left(t_{0}\right)=$ $x_{0}$. Then for almost all $t \in\left[t_{0}, T\right]$, one has

$$
\left\{\begin{array}{l}
\dot{x}_{1}(t) \in f\left(t, x_{1}(t)\right)-A_{t, x_{1}(t)}\left(x_{1}(t)\right), \\
\dot{x}_{2}(t) \in f\left(t, x_{2}(t)\right)-A_{t, x_{2}(t)}\left(x_{2}(t)\right) .
\end{array}\right.
$$

Using the hypo-monotonicity of $A$ and Lipschitz continuity of $f$, we have

$$
\begin{aligned}
\left\langle\dot{x}_{1}(t)-\dot{x}_{2}(t), x_{1}(t)-x_{2}(t)\right\rangle & \leq\left\langle f\left(t, x_{1}(t)\right)-f\left(t, x_{2}(t)\right), x_{1}(t)-x_{2}(t)\right\rangle+k_{M}\left\|x_{1}(t)-x_{2}(t)\right\|^{2} \\
& \leq\left(l_{M}+k_{M}\right)\left\|x_{1}(t)-x_{2}(t)\right\|^{2}
\end{aligned}
$$

where $k_{M}, l_{M}$ are defined in (33) and $M>0$ is a constant such that

$$
\max \left\{\sup _{t \in\left[t_{0}, T\right]}\left\|x_{1}(t)\right\|, \sup _{t \in\left[t_{0}, T\right]}\left\|x_{2}(t)\right\|\right\} \leq M .
$$

Then we have

$$
\frac{d}{d t}\left\|x_{1}(t)-x_{2}(t)\right\|^{2} \leq 2\left(l_{M}+k_{M}\right)\left\|x_{1}(t)-x_{2}(t)\right\|^{2} \quad \text { a.e. } t \in\left[t_{0}, T\right],
$$

and we obtain the conclusion by using the continuous Gronwall's inequality in Lemma 2.6.

Remark 3.2 Let us provide some cases such that $A$ is hypo-monotone.

(i) Clearly if $A$ dependent only on the time, i.e. $A_{t, x} \equiv A_{t}$, then $A$ is hypo-monotone.

(ii) We show that the property also holds if $A_{t, x}(\cdot)=B_{t}(\cdot+\alpha x)$ where $\alpha>-1$ and $B_{t}$ is a maximal monotone operator for each $t \geq 0$. By using Minty's theorem, it is easy to see that $A_{t, x}$ is maximal monotone. In addition, for all $t \in[0, T], x_{i} \in \mathbb{R}^{n}, x_{i}^{*} \in A_{t, x_{i}}\left(x_{i}\right), i=1,2$ we have

$$
\left\langle x_{1}^{*}-x_{2}^{*}, x_{1}-x_{2}\right\rangle=\frac{1}{1+\alpha}\left\langle x_{1}^{*}-x_{2}^{*},(\alpha+1)\left(x_{1}-x_{2}\right)\right\rangle \geq 0 .
$$


(iii) If $A_{t, x}=\left(B_{t, x}^{-1}+D\right)^{-1}$ where $D$ is a positive semidefinite matrix, $B_{t, x}(\cdot)=C_{t}(\cdot+g(t, x))$ and $C_{t}: \mathbb{R}^{n} \rightrightarrows \mathbb{R}^{n}$ is a maximal monotone operator for each $t \in[0, T]$ and $g:[0, T] \times \mathbb{R}^{n} \rightarrow \operatorname{rge}\left(D+D^{T}\right)$ is a Lipschitz continuous in bounded sets w.r.t the second variable. This particular form of $A$ appears widely in Lur'e dynamical systems (see, e.g., [1, 8, [9, 10, 28]). Let us first show that $B_{t, x}$ is a maximal monotone operator and then so is $A_{t, x}$ since $D$ is a maximal monotone operator with full domain.

- Monotonicity: Let $y_{i}^{*} \in B_{t, x}\left(y_{i}\right)=C_{t}\left(y_{i}+g(t, x)\right), i=1,2$. Then

$$
\left\langle y_{1}^{*}-y_{2}^{*}, y_{1}-y_{2}\right\rangle=\left\langle y_{1}^{*}-y_{2}^{*},\left(y_{1}+g(t, x)\right)-\left(y_{2}+g(t, x)\right)\right\rangle \geq 0 .
$$

- Maximality: It is equivalent to show that $B_{t, x}^{-1}$ is maximal. Let $\left(y, y^{*}\right) \in \mathbb{R}^{2 n}$, suppose that

$$
\left\langle y^{*}-z^{*}, y-z\right\rangle \geq 0,
$$

for all $\left(z, z^{*}\right)$ satisfying $z \in B_{t, x}^{-1}\left(z^{*}\right) \Leftrightarrow z^{*} \in C_{t}(z+g(t, x)) \Leftrightarrow z+g(t, x) \in C_{t}^{-1}\left(z^{*}\right)$. We want to prove that $y \in B_{t, x}^{-1}\left(y^{*}\right)$. Indeed, we have

$$
\left\langle y^{*}-z^{*},(y+g(t, x))-(z+g(t, x))\right\rangle \geq 0 .
$$

Since $C_{t}^{-1}$ is maximal monotone, we must have $\left.y+g(t, x)\right) \in C_{t}^{-1}\left(y^{*}\right)$, or equivalently, $y \in$ $B_{t, x}^{-1}\left(y^{*}\right)$. Consequently we obtain the maximality of $B_{t, x}$.

It remains to check that $A$ is hypo-monotone. For all $t \in[0, T], x_{i} \in \mathbb{R}^{n} \cap M \mathbb{B}, x_{i}^{*} \in A_{t, x_{i}}\left(x_{i}\right)$, $i=1,2$ we have

$$
\begin{aligned}
& x_{i}^{*} \in A_{t, x_{i}}\left(x_{i}\right)=\left(B_{t, x_{i}}^{-1}+D\right)^{-1}\left(x_{i}\right) \\
\Leftrightarrow \quad & x_{i}^{*} \in B_{t, x_{i}}\left(x_{i}-D x_{i}^{*}\right)=C_{t}\left(x_{i}-D x_{i}^{*}+g\left(t, x_{i}\right)\right) .
\end{aligned}
$$

From the monotonicity of $C_{t}$, we have

$$
\begin{aligned}
\left\langle x_{1}^{*}-x_{2}^{*}, x_{1}-x_{2}\right\rangle & \geq\left\langle D\left(x_{1}^{*}-x_{2}^{*}\right), x_{1}^{*}-x_{2}^{*}\right\rangle-\left\langle x_{1}^{*}-x_{2}^{*}, g\left(t, x_{1}\right)-g\left(t, x_{2}\right)\right\rangle \\
& \geq c_{1}\left\|x_{1}^{* i m}-x_{2}^{* i m}\right\|^{2}-L_{g}\left\|x_{1}^{* i m}-x_{2}^{* i m}\right\|\left\|x_{1}-x_{2}\right\|\left(\text { since } \operatorname{rge}(g) \subset \operatorname{rge}\left(D+D^{T}\right)\right) \\
& \left.\geq-\frac{L_{g}^{2}}{4 c_{1}}\left\|x_{1}-x_{2}\right\|^{2} \text { (using the inequality } a^{2}+b^{2} \geq 2 a b, a, b \in \mathbb{R}\right)
\end{aligned}
$$

where $L_{g}$ is the Lipschitz constant of $g$ w.r.t the second variable in $M \mathbb{B}, c_{1}$ is defined in Lemma[2.1 and $x^{* i m}$ denotes the projection of $x^{*}$ onto $\operatorname{rge}\left(D+D^{T}\right)$. Consequently, the conclusion follows.

\section{$4 \quad$ Stability analysis by using nonsmooth Lyapunov pairs}

In this section, we want to provide a characterization for lower semi-continuous Lyapunov pairs associated with problem (1) by using proximal analysis. From here, we suppose that all assumptions of Theorem 3.2 are satisfied, then for each $\left(t_{0}, x_{0}\right) \in \mathcal{A}_{1}$, the problem (1) has a unique solution $x(\cdot)$ defined on $\left[t_{0},+\infty\right)$. Next we recall the definition of a Lyapunov pair associated with problem (1). Denote by

$$
\Gamma\left([0,+\infty) \times \mathbb{R}^{n}\right):=\left\{\varphi:[0,+\infty) \times \mathbb{R}^{n} \rightarrow \mathbb{R} \cup\{+\infty\} \mid \varphi \text { is proper and lsc }\right\},
$$

and

$$
\Gamma_{+}\left([0,+\infty) \times \mathbb{R}^{n}\right):=\left\{\varphi:[0,+\infty) \times \mathbb{R}^{n} \rightarrow \mathbb{R}_{+} \cup\{+\infty\} \mid \varphi \text { is proper and lsc }\right\}
$$


Definition 4.1 Let $V \in \Gamma\left([0,+\infty) \times \mathbb{R}^{n}\right), W \in \Gamma_{+}\left([0,+\infty) \times \mathbb{R}^{n}\right)$ and $a \geq 0$. We say that $(V, W)$ is an a-Lyapunov pair for problem (1) if for all $\left(t_{0}, x_{0}\right) \in \mathcal{A}_{1}$ we have

$$
e^{a\left(t-t_{0}\right)} V\left(t, x\left(t ; t_{0} ; x_{0}\right)\right)+\int_{t_{0}}^{t} W\left(\tau, x\left(\tau ; t_{0} ; x_{0}\right)\right) d \tau \leq V\left(t_{0}, x_{0}\right) \text { for all } t \geq t_{0} \geq 0,
$$

where $x\left(t ; t_{0}, x_{0}\right)$ denotes the unique solution of problem (1) satisfying $x\left(t_{0}\right)=x_{0}$. If $a=0$, then $(V, W)$ is called a Lyapunov pair. In addition, $V$ is called a Lyapunov function if $W=0$.

Remark 4.1 (i) Note that if the uniqueness is not available, we can deal with the weak Lyapunov pairs by using similar arguments, i.e., the inequality (36) is satisfied for at least one trajectory of problem (1).

(ii) Let $x(\cdot):=x\left(\cdot ; t_{0}, x_{0}\right)$. From Theorem 3.1, we know that

$$
\|x(t)\| \leq\left\|x_{0}\right\|+m\left(x_{0}\right), \quad \forall t \in\left[t_{0}, t_{0}+1\right],
$$

where $m\left(x_{0}\right)$ is defined in (32). Combining with Assumptions 1.2 and 2, we have

$$
\begin{aligned}
\left\|\left(f(t, x(t))-A_{t, x(t)}(x(t))\right)^{0}\right\| & \leq c_{f}(1+\|x(t)\|)+c_{A}(1+2\|x(t)\|) \\
& \leq c_{f}+c_{A}+\left(c_{f}+2 c_{A}\right)\left(\left\|x_{0}\right\|+m\left(x_{0}\right)\right) .
\end{aligned}
$$

Let

$$
M\left(x_{0}\right):=c_{f}+c_{A}+\left(c_{f}+2 c_{A}+1\right)\left(\left\|x_{0}\right\|+m\left(x_{0}\right)\right) .
$$

Then $M\left(x_{0}\right)>m\left(x_{0}\right),\left(f(t, x(t))-A_{t, x(t)}(x(t))\right) \cap M\left(x_{0}\right) \mathbb{B} \neq \emptyset$ for all $t \in\left[t_{0}, t_{0}+1\right]$ and $M(\cdot)$ is a continuous function w.r.t $x_{0}$.

Lemma 4.1 Let $x(\cdot):=x\left(\cdot ; t_{0}, x_{0}\right)$. We define the mapping $Z:\left[t_{0}, t_{0}+1\right] \rightrightarrows \mathbb{R}^{n}$ as follows

$$
Z(t):=\left(f(t, x(t))-A_{t, x(t)}(x(t))\right) \cap M\left(x_{0}\right) \mathbb{B},
$$

where $M\left(x_{0}\right)$ is defined in (37). Then $Z$ has non-empty, convex compact values with closed graph and uniformly bounded. In particular, $Z$ is upper semi-continuous, i.e., given $t_{1} \in\left[t_{0}, T\right]$, for any $\varepsilon>0$, we can find $\delta>0$ such that

$$
Z(t) \subset Z\left(t_{1}\right)+\varepsilon \mathbb{B}, \text { for }\left|t-t_{1}\right|<\delta .
$$

Proof. Obviously, $Z$ has non-empty, convex compact values and uniformly bounded. It remain to check that the graph of $Z$ is closed, i.e., if $y_{n} \in Z\left(t_{n}\right)$ and $y_{n} \rightarrow y, t_{n} \rightarrow t$, we must have $y \in Z(t)$. First we have $y \in M\left(x_{0}\right) \mathbb{B}$. Let $B_{n}:=A_{t_{n}, x\left(t_{n}\right)}, B=A_{t, x(t)}$ then $B_{n}, B$ are maximal monotone operators and

$$
\operatorname{dis}\left(B_{n}, B\right)=\operatorname{dis}\left(A_{t_{n}, x\left(t_{n}\right)}, A_{t, x(t)}\right) \leq L_{1}\left|t_{n}-t\right|+L_{2}\left\|x\left(t_{n}\right)-x(t)\right\| \rightarrow 0, \text { as } n \rightarrow+\infty .
$$

On the other hand, we have

$$
f\left(t_{n}, x\left(t_{n}\right)\right)-y_{n} \in B_{n}\left(x\left(t_{n}\right)\right), \quad f\left(t_{n}, x\left(t_{n}\right)\right)-y_{n} \rightarrow f(t, x(t))-y \quad \text { and } x\left(t_{n}\right) \rightarrow x(t) .
$$

Using Lemma 2.4. one obtains that $f(t, x(t))-y \in B(x(t))$, or equivalently $y \in f(t, x(t))-$ $A_{t, x(t)}(x(t))$. Consequently $y \in Z(t)$ and we have the closedness of the graph of $Z$. Then classically, one has the upper semi-continuity of $Z$ (see, e.g., [11]). 
Lemma 4.2 Let $x(\cdot):=x\left(\cdot ; t_{0}, x_{0}\right)$. There exists a sequence a sequence $\left(t_{n}\right)$ such that $t_{n} \rightarrow t_{0}^{+}$and

$$
\lim _{n \rightarrow+\infty} \frac{x\left(t_{n}\right)-x\left(t_{0}\right)}{t_{n}-t_{0}}=v \in Z\left(t_{0}\right)
$$

where the mapping $Z$ is defined in Lemma 4.1 .

Proof. Let

$$
v(t):=\frac{x(t)-x\left(t_{0}\right)}{t-t_{0}}, t>t_{0} .
$$

Then $v(t)$ is bounded by $m\left(x_{0}\right)$ (Theorem 3.1) for all $t \in\left[t_{0}, t_{0}+1\right]$. Hence there exist a sequence $\left(t_{n}\right)$ and $v \in \mathbb{R}^{n}$ such that $t_{n} \rightarrow t_{0}^{+}$and $v_{n}:=v\left(t_{n}\right)$ converges to $v$. Let be given $\varepsilon>0$. Note that for $n$ large enough, by using Lemma 4.1 , we have

$$
x^{\prime}(s) \in Z(s) \subset Z\left(t_{0}\right)+\varepsilon \mathbb{B} \text {, a.e. } s \in\left[t_{0}, t_{n}\right] .
$$

Thus

$$
v_{n}=\frac{1}{t_{n}-t_{0}} \int_{t_{0}}^{t_{n}} x^{\prime}(s) d s \in Z\left(t_{0}\right)+\varepsilon \mathbb{B},
$$

which implies that $v \in Z\left(t_{0}\right)+\varepsilon \mathbb{B}$. Since $\varepsilon$ is arbitrary, we deduce that $v \in Z\left(t_{0}\right)$ and the conclusion follows.

The following result provides necessary and sufficient conditions for a Lyapunov pair associated with problem (1).

Theorem 4.1 Let $V \in \Gamma\left([0, T] \times \mathbb{R}^{n}\right), W \in \Gamma_{+}\left([0, T] \times \mathbb{R}^{n}\right), a \geq 0$ and $\operatorname{dom}(V) \subset \mathcal{A}_{1}$. Then the following statements are equivalent:

(i) For each $\left(t_{0}, x_{0}\right) \in \operatorname{dom}(V)$, we have

$$
e^{a\left(t-t_{0}\right)} V(t, x(t))+\int_{t_{0}}^{t} W(\tau, x(\tau)) d \tau \leq V\left(t_{0}, x_{0}\right) \quad \forall t \geq t_{0},
$$

where $x(\cdot):=x\left(\cdot ; t_{0}, x_{0}\right)$.

(ii) For each $\left(t_{0}, x_{0}\right) \in \operatorname{dom}(V)$ and $(\theta, \xi) \in \partial^{P} V\left(t_{0}, x_{0}\right)$, we have

$$
\theta+\underset{v \in\left(f\left(t_{0}, x_{0}\right)-A_{t_{0}, x_{0}}\left(x_{0}\right)\right) \cap M\left(x_{0}\right) \mathbb{B}}{\min }\langle\xi, v\rangle+a V\left(t_{0}, x_{0}\right)+W\left(t_{0}, x_{0}\right) \leq 0 .
$$

(iii) For each $\left(t_{0}, x_{0}\right) \in \operatorname{dom}(V)$, we have

$$
\left\{\begin{array}{c}
\sup _{(\theta, \xi) \in \partial^{P} V\left(t_{0}, x_{0}\right)}\left\{\theta+\min _{v \in\left(f\left(t_{0}, x_{0}\right)-A_{t_{0}, x_{0}}(x)\right) \cap M\left(x_{0}\right) \mathbb{B}}\langle\xi, v\rangle+a V\left(t_{0}, x_{0}\right)+W\left(t_{0}, x_{0}\right)\right\} \leq 0, \\
\sup _{(\theta, \xi) \in \partial^{\infty} V\left(t_{0}, x_{0}\right)}\left\{\theta+\min _{v \in\left(f(t, x)-A_{t_{0}, x_{0}}(x)\right) \cap M\left(x_{0}\right) \mathbb{B}}\langle\xi, v\rangle\right\} \leq 0 .
\end{array}\right.
$$

(iv) For each $\left(t_{0}, x_{0}\right) \in \operatorname{dom}(V)$, for any $M>0$ large enough, we have

$$
\left\{\begin{array}{l}
\sup _{\left.(\theta, \xi) \in \partial^{P} V t_{0}, x_{0}\right)}\left\{\theta+\min _{v \in\left(f\left(t_{0}, x_{0}\right)-A_{t_{0}, x_{0}}(x)\right) \cap M \mathbb{B}}\langle\xi, v\rangle+a V\left(t_{0}, x_{0}\right)+W\left(t_{0}, x_{0}\right)\right\} \leq 0, \\
\sup _{(\theta, \xi) \in \partial^{\infty} V\left(t_{0}, x_{0}\right)}\left\{\theta+\min _{v \in\left(f\left(t_{0}, x_{0}\right)-A_{t_{0}, x_{0}}(x)\right) \cap M \mathbb{B}}\langle\xi, v\rangle\right\} \leq 0 .
\end{array}\right.
$$

where $M\left(x_{0}\right)$ is defined in (37). 
Proof. Without loss of generality, suppose that $W$ is Lipschitz continuous on bounded sets (see [2, Lemma 3.1] or [11]). The plan of the proof is the following: $(i) \Rightarrow(i i) \Rightarrow(i i i) \Rightarrow(i v) \Rightarrow(i)$.

$(i) \Rightarrow(i i):$ Let $\left(t_{0}, x_{0}\right) \in \operatorname{dom}(V)$ and $(\theta, \xi) \in \partial^{P} V\left(t_{0}, x_{0}\right)$. Then $(\theta, \xi,-1) \in N_{\text {epi } V}^{P}\left(\left(t_{0}, x_{0}\right), V\left(t_{0}, x_{0}\right)\right)$. Let $x(\cdot):=x\left(\cdot ; t_{0}, x_{0}\right)$. From $(i)$, one obtains that

$$
\left((t, x(t)), e^{-a\left(t-t_{0}\right)} V\left(t_{0}, x_{0}\right)-e^{-a\left(t-t_{0}\right)} \int_{t_{0}}^{t} W(\tau, x(\tau)) d \tau\right) \in \text { epi } V \quad \forall t \geq t_{0}
$$

By the definition of $N_{\text {epi } V}^{P}(y, V(y))$, there exists $\beta>0$ such that for all $t \geq t_{0}$, one has

$$
\begin{aligned}
& \left\langle(\theta, \xi,-1),\left((t, x(t)), e^{-a\left(t-t_{0}\right)} V\left(t_{0}, x_{0}\right)-e^{-a\left(t-t_{0}\right)} \int_{t_{0}}^{t} W(\tau, x(\tau)) d \tau\right)-\left(\left(t_{0}, x_{0}\right), V\left(t_{0}, x_{0}\right)\right)\right\rangle \\
\leq & \beta\left\|\left((t, x(t)), e^{-a\left(t-t_{0}\right)} V\left(t_{0}, x_{0}\right)-e^{-a\left(t-t_{0}\right)} \int_{t_{0}}^{t} W(\tau, x(\tau)) d \tau\right)-\left(\left(t_{0}, x_{0}\right), V\left(t_{0}, x_{0}\right)\right)\right\|^{2},
\end{aligned}
$$

which is equivalent to

$$
\left\langle\theta, t-t_{0}\right\rangle+\left\langle\xi, x(t)-x_{0}\right\rangle-R(t) \leq \beta\left(t-t_{0}\right)^{2}+\beta\left\|x(t)-x_{0}\right\|^{2}+\beta R^{2}(t),
$$

where

$$
R(t):=\left(e^{-a\left(t-t_{0}\right)}-1\right) V\left(t_{0}, x_{0}\right)-e^{-a\left(t-t_{0}\right)} \int_{t_{0}}^{t} W(\tau, x(\tau)) d \tau .
$$

Using Lemma 4.2 , there exists a sequence $\left(t_{n}\right)$ such that $t_{n} \rightarrow t_{0}^{+}$and

$$
\lim _{n \rightarrow+\infty} \frac{x\left(t_{n}\right)-x_{0}}{t_{n}-t_{0}}=v
$$

exists and $\left.v \in f\left(t_{0}, x_{0}\right)-A_{t_{0}, x_{0}}\left(x_{0}\right)\right) \cap M\left(x_{0}\right) \mathbb{B}$.

Taking $t=t_{n}$ in (45). Dividing both sides of (45) by $t_{n}-t_{0}>0$, letting $n \rightarrow+\infty$, one obtains

$$
\theta+\langle\xi, v\rangle+a V\left(t_{0}, x_{0}\right)+W\left(t_{0}, x_{0}\right) \leq 0 .
$$

Therefore, we obtain (ii).

$(i i) \Rightarrow(i i i)$ : It remains to check the second inequality of $(i i i)$. Let $(\theta, \xi) \in \partial^{\infty} V\left(t_{0}, x_{0}\right)$. Then there exist sequences $\left(\alpha_{k}\right)_{k \in \mathbb{N}} \subset \mathbb{R}_{+},\left(t_{k}, x_{k}\right)_{k \in \mathbb{N}},\left(\theta_{k}, \xi_{k}\right)_{k \in \mathbb{N}}$ such that $\alpha_{k} \rightarrow 0^{+},\left(t_{k}, x_{k}\right) \rightarrow_{V}\left(t_{0}, x_{0}\right)$, $\left(\theta_{k}, \xi_{k}\right) \in \partial^{P} V\left(t_{k}, x_{k}\right)$ and $\alpha_{k}\left(\theta_{k}, \xi_{k}\right) \rightarrow(\theta, \xi)$. For each $k$, one can find $v_{k} \in\left(f\left(t_{k}, x_{k}\right)-A_{t_{k}, x_{k}}\left(x_{k}\right)\right) \cap$ $M\left(x_{k}\right) \mathbb{B}$ such that

$$
\theta_{k}+\left\langle\xi_{k}, v_{k}\right\rangle+a V\left(y_{k}\right)+W\left(y_{k}\right) \leq 0 .
$$

Given $\varepsilon>0$, for $k$ large enough, we have $v_{k} \in\left(-f\left(t_{k}, x_{k}\right)-A_{t_{k}, x_{k}}\left(x_{k}\right)\right) \cap\left(M\left(x_{0}\right)+\varepsilon\right) \mathbb{B}$. Since the sequence $\left(v_{k}\right)$ is bounded, one can extract a subsequence, without relabelling, and some $v$ such that $v_{k} \rightarrow v$ and $v \in\left(-f\left(t_{0}, x_{0}\right)-A_{t_{0}, x_{0}}\left(x_{0}\right)\right) \cap\left(M\left(x_{0}\right)+\varepsilon\right) \mathbb{B}$. Since $\varepsilon$ is arbitrary, we have $v \in\left(-f\left(t_{0}, x_{0}\right)-A_{t_{0}, x_{0}}\left(x_{0}\right)\right) \cap M\left(x_{0}\right) \mathbb{B}$. Multiplying both sides of 46 by $\alpha_{k}$ and let $k \rightarrow+\infty$ then one obtains that

$$
\theta+\langle\xi, v\rangle \leq 0
$$

which implies the second inequality of (iii). 
$($ iii $) \Rightarrow(i v)$ : Obviously for any $M>M\left(x_{0}\right)$.

$(i v) \Rightarrow(i):$ Let $\left(t_{0}, x_{0}\right) \in \operatorname{dom} V$. Let $x(\cdot)=x\left(\cdot ; t_{0}, x_{0}\right)$ be the solution of (1) with $x\left(t_{0}\right)=x_{0}$. Given any $T>0$, we define the functions $h:\left[t_{0}, T\right] \rightarrow \mathbb{R}_{+}, \gamma:\left[t_{0}, T\right] \rightarrow \mathbb{R}, z:\left[t_{0}, T\right] \rightarrow\left[t_{0}, T\right] \times \mathbb{R}^{n} \times \mathbb{R}$ and $\eta:\left[t_{0}, T\right] \rightarrow \mathbb{R}_{+}$as follows

$$
\begin{gathered}
h(t):=\int_{t_{0}}^{t} W\left(\tau, x\left(\tau ; x_{0}\right)\right) d \tau, \gamma(t):=e^{-a\left(t-t_{0}\right)}\left(V\left(t_{0}, x_{0}\right)-h(t)\right), \\
z(t):=\left(t, x\left(t ; x_{0}\right), \gamma(t)\right), \quad \eta(t):=\frac{1}{2} d^{2}(z(t), \text { epi } V) .
\end{gathered}
$$

As in [2], $\eta$ is Lipschitz continuous on every compact interval in $\left(t_{0}, T\right)$ and for all $t \in\left(t_{0}, T\right)$, one has

$$
\partial^{C} \eta(t)=d(z(t) \text {, epi } V) \partial^{C} d(z(\cdot), \text { epi } V)(t) \neq \emptyset,
$$

where $\partial^{C}$ denotes the Clarke subdifferential. We have then an estimation of $\partial^{C} \eta$ as in Lemma 4.3 . Let $t_{0}<s \leq t<T$. By using Gronwall's inequality one has

$$
e^{-N t} \eta(t) \leq e^{-N s} \eta(s),
$$

where $N>0$ is defined in Lemma 4.3 . Let $s \rightarrow t_{0}$ then one has $d(z(t)$, epi $V)=0$ which implies that

$$
e^{a\left(t-t_{0}\right)} V(t, x(t))+\int_{t_{0}}^{t} W(\tau, x(\tau)) d \tau \leq V\left(t_{0}, x_{0}\right), \quad \forall t_{0}<t<T .
$$

Since $T$ is arbitrary, we obtain the conclusion.

The following lemma can be used to deduce $(i v) \Rightarrow(i)$ in the proof of Theorem 4.1 .

Lemma 4.3 We can find some $N>0$ such that for almost all $t \in\left(t_{0}, T\right)$, one has

$$
\partial^{C} \eta(t) \subset(-\infty, N \eta(t)]
$$

Proof. Let $t \in\left(t_{0}, T\right)$ such that $x(\cdot)$ is differentiable at $t$. If $z(t) \in$ epi $V$, then $\partial^{C} \eta(t)=\{0\}$ and the conclusion holds. Otherwise, assume that $z(t) \notin$ epi $V$. By using [2, Lemma A.3] and noting that $\dot{x}(t) \in f(t, x(t))-A_{t, x(t)}(x(t))$, we obtain

$$
\partial^{C} \eta(t) \subset \overline{\mathrm{co}}\left[\bigcup_{(s, u, \mu) \in \mathcal{M}}\left\langle z(t)-\left(\begin{array}{c}
s \\
u \\
\mu
\end{array}\right),\left(\begin{array}{c}
1 \\
\left.-f(t, x(t))-A_{t, x(t)}(x(t))\right) \\
-a \gamma(t)-e^{-a t} W(t, x(t))
\end{array}\right)\right],\right.
$$

where $\mathcal{M}:=\operatorname{Proj}(z(t)$, epi $V)$. Then it is sufficient to prove that for all $(s, u, \mu) \in \mathcal{M}$ and $\forall x^{*} \in$ $\left(f(t, x(t))-A_{t, x(t)}(x(t))\right)$, we have

$$
\left\langle z(t)-\left(\begin{array}{c}
s \\
u \\
\mu
\end{array}\right),\left(\begin{array}{c}
1 \\
x^{*} \\
-a \gamma(t)-e^{-a\left(t-t_{0}\right)} W(t, x(t))
\end{array}\right)\right\rangle \leq N \eta(t),
$$


for some $N>0$. Since $(s, u, \mu) \in \operatorname{Proj}(z(t)$, epi $V)$, the vector $z(t)-(s, u, \mu)=(t-s, x(t)-u, \gamma(t)-$ $\mu) \in N_{\text {epi } V}^{P}(s, u, \mu)$. Hence we have $\gamma(t)-\mu \leq 0$. If $\gamma(t)-\mu=0$ then $(t-s, x(t)-u) \in \partial^{\infty} V(s, u)$ and if $\gamma(t)-\mu<0$ then $\left(\frac{t-s}{\mu-\gamma(t)}, \frac{x(t)-u}{\mu-\gamma(t)}\right) \in \partial^{P} V(s, u)$. From $\left.i v\right)$, there exists $v \in\left(f(s, u)-A_{s, u}(u)\right)$ such that

$$
t-s+\langle x(t)-u, v\rangle \leq(\gamma(t)-\mu)(a V(s, u)+W(s, u)) .
$$

On the other hand, we have $\sup _{t \in\left[t_{0}, T\right]}\|x(t)\| \leq M_{1}$ for some $M_{1}>0$. From the fact that $(s, u, \mu) \in$ $\operatorname{Proj}(z(t)$, epi $V)$ and $z\left(t_{0}\right) \in$ epi $V$, we have

$$
\|z(t)-(s, u, \mu)\| \leq\|z(t)-z(0)\|
$$

which implies that

$$
\|u\| \leq\|(s, u, \mu)\| \leq 2\|z(t)\|+\|z(0)\| .
$$

Since $z(t)$ is uniformly bounded, one can find some $M_{2}>M_{1}$ such that $u \in M_{2} \mathbb{B}$. Thanks to (50), the hypo-monotonicity of $A$ and the Lipschitz continuous of $f$ on $M_{2} \mathbb{B}$, one has

$$
\begin{aligned}
t-s+\left\langle x(t)-u, x^{*}\right\rangle & =t-s+\left\langle x(t)-u, x^{*}-v+v\right\rangle \\
& \leq\left(l_{M_{2}}+k_{M_{2}}\right)\|x(t)-u\|^{2}+(\gamma(t)-\mu)(a V(s, u)+W(s, u)),
\end{aligned}
$$

where $k_{M_{2}}$ and $l_{M_{2}}$ are defined in (33). Note that we already have $\gamma(t)-\mu \leq 0$. If $\gamma(t)-\mu<0$ and suppose that $V(s, u) \leq \gamma(t)$. One obtains a contradiction

$$
d(z(t), \operatorname{epi} V) \leq d(z(t),(s, u, \gamma(t)))<d(z(t),(s, u, \mu))=d(z(t), \operatorname{epi} V) .
$$

Hence if $\gamma(t)-\mu<0$, we must have $V(s, u)>\gamma(t)$. Therefore, we always obtain

$$
(\mu-\gamma(t))(\gamma(t)-V(s, u)) \leq 0 .
$$

Consequently,

$$
\begin{aligned}
& t-s+\left\langle x(t)-u, x^{*}\right\rangle+a(\mu-\gamma(t)) \gamma(t)+(\mu-\gamma(t)) e^{-a\left(t-t_{0}\right)} W(t, x(t)) \\
& \leq\left(l_{M_{2}}+k_{M_{2}}\right)\|x(t)-u\|^{2}+(\gamma(t)-\mu)(a V(s, u)+W(s, u)) \\
& +a(\mu-\gamma(t)) \gamma(t)+(\mu-\gamma(t)) e^{-a\left(t-t_{0}\right)} W(t, x(t)) \\
& \leq\left(l_{M_{2}}+k_{M_{2}}\right)\|x(t)-u\|^{2}+a(\mu-\gamma(t))(\gamma(t)-V(s, u))+(\mu-\gamma(t))(W(t, x(t))-W(s, u)) \\
& \leq\left(l_{M_{2}}+k_{M_{2}}\right)\|x(t)-u\|^{2}+L_{W}|\mu-\gamma(t)|(|t-s|+\|x(t)-u\|) \leq\left(\frac{L_{W}}{2}+l_{M_{2}}+k_{M_{2}}\right) \eta(t),
\end{aligned}
$$

where $L_{W}$ is the Lipschitz constant of $W$ on $[0, T] \times M_{2} \mathbb{B}$. Therefore Lemma 4.3 holds with $N:=\frac{L_{W}}{2}+l_{M_{2}}+k_{M_{2}}$.

Now let us provide some illustrative examples as follows.

Example 4.1 Let be given $p>0$ and a differentiable function $g:[0,+\infty) \rightarrow \mathbb{R}$ such that $\dot{g} \leq 2 g$. We consider the following systems in $\mathbb{R}^{3}$ :

$$
\left\{\begin{array}{l}
\dot{x}_{1}=-x_{1}-g(t) x_{2}, \\
\dot{x}_{2}=x_{1}-x_{2}, \\
\dot{x}_{3} \in-\operatorname{Sign}\left(x_{3}\right)+p\left|x_{3}\right| .
\end{array}\right.
$$


Then we can reduce the inclusion (51) into the our problem (1) as follows

$$
\dot{x} \in f(t, x)-A_{t, x}(x),
$$

where

$$
f(t, x)=\left(\begin{array}{c}
-x_{1}-g(t) x_{2} \\
x_{1}-x_{2} \\
p\left|x_{3}\right|
\end{array}\right),
$$

and

$$
A_{t, y}(x)=\left(\begin{array}{c}
0 \\
0 \\
-\operatorname{Sign}\left(x_{3}\right)
\end{array}\right) .
$$

Then all assumptions of Theorem 3.2 are satisfied. Consequently, for each initial condition, the system (51) has a unique solution. Note that for each $(t, x) \in[0,+\infty) \times \mathbb{R}^{2 n}$, we can find some $M>0$ such that $f(t, x)-A_{t, x}(x) \in M \mathbb{B}$ and hence $\left(f(t, x)-A_{t, x}(x)\right) \cap M \mathbb{B}=f(t, x)-A_{t, x}(x)$. Let

$$
V(t, x)=\left\{\begin{array}{l}
x_{1}^{2}+(1+g(t)) x_{2}^{2}+\left|x_{3}\right|, \text { if } x_{3} \leq \frac{1}{p}, \\
+\infty, \text { if } x_{3}>\frac{1}{p} .
\end{array}\right.
$$

Then $V$ is a lower semi-continuous function and

$$
\partial^{P} V(t, x)=\left(\begin{array}{c}
\dot{g}(t) x_{2}^{2} \\
2 x_{1} \\
2 x_{2}(1+g(t)) \\
\xi\left(x_{3}\right)
\end{array}\right),
$$

where

$$
\xi\left(x_{3}\right):= \begin{cases}\operatorname{Sign}\left(x_{3}\right) & \text { if } x_{3}<\frac{1}{p}, \\ 1+\mathbb{R}^{+} & \text {if } x_{3}=\frac{1}{p} \\ \emptyset & \text { if } x_{3}>\frac{1}{p} .\end{cases}
$$

For all $(t, x) \in \operatorname{dom}(V)$ and $(\theta, \xi) \in \partial^{P} V(t, x)$ we have

$$
\begin{aligned}
& \theta+\min _{v \in\left(f(t, x)-A_{t, x}(x)\right)}\langle\xi, v\rangle \\
= & \dot{g}(t) x_{2}^{2}+2 x_{1}\left(-x_{1}-g(t) x_{2}\right)+2 x_{2}(1+g(t))\left(x_{1}-x_{2}\right)+\operatorname{Sign}\left(x_{3}\right)\left(-\operatorname{Sign}\left(x_{3}\right)+p\left|x_{3}\right|\right) \\
= & -2 x_{1}^{2}+2 x_{1} x_{2}-2 x_{2}^{2}+x_{2}^{2}(\dot{g}(t)-2 g(t))+\operatorname{Sign}^{2}\left(x_{3}\right)\left(p x_{3}-1\right) \\
\leq & -x_{1}^{2}-x_{2}^{2}-\left(x_{1}-x_{2}\right)^{2} \leq 0 .
\end{aligned}
$$

Hence, $V$ is a Lyapunov function for (51) by using Theorem 4.1. 
Example 4.2 Let be given $\alpha>0, \gamma>0$ and $\beta \in \mathbb{R}$. We consider the following differential inclusion in $\mathbb{R}^{2}$ :

$$
\left\{\begin{array}{l}
\dot{x}_{1} \in-\alpha x_{1}+\beta x_{2}-N_{C\left(t, x_{1}\right)}\left(x_{1}\right), \\
\dot{x}_{2} \in-\beta x_{1}+x_{2}-\gamma \operatorname{Sign}\left(x_{2}\right), \\
x(0)=\left(x_{01} x_{02}\right)^{T}
\end{array}\right.
$$

where $C\left(t, x_{1}\right):=\left[-\left(t+2\left|x_{01}\right|\right), t+2\left|x_{01}\right|\right]+x_{1} / 2$. Then we can rewrite (53) into our form (1) as follows

$$
\dot{x} \in f(t, x)-A_{t, x}(x),
$$

where

$$
f(t, x)=\left(\begin{array}{c}
-\alpha x_{1}+\beta x_{2} \\
-\beta x_{1}+x_{2}
\end{array}\right),
$$

and

$$
A_{t, x}(x)=\left(\begin{array}{c}
-N_{C\left(t, x_{1}\right)}\left(x_{1}\right) \\
-\gamma \operatorname{Sign}\left(x_{2}\right)
\end{array}\right) .
$$

Then it is easy to see that $x_{01} \in C\left(0, x_{01}\right)$ and all assumptions of Theorem 3.2 are satisfied. Let us consider the function $V$ as follows

$$
V(t, x)=\left\{\begin{array}{l}
\frac{1}{2} x_{1}^{2}+\frac{1}{2} x_{2}^{2}, \text { if }\left|x_{2}\right| \leq \gamma \\
+\infty, \text { if } x_{2}>\gamma
\end{array}\right.
$$

Then $V$ is a lower semi-continuous function and

$$
\partial^{P} V(t, x)=\left(\begin{array}{c}
0 \\
x_{1} \\
\xi\left(x_{2}\right)
\end{array}\right)
$$

where

$$
\xi\left(x_{2}\right):=\left\{\begin{array}{l}
x_{2} \text { if }-\gamma<x_{2}<\gamma, \\
\left\{k x_{2}: k \geq 1\right\} \text { if }\left|x_{2}\right|=\gamma, \\
\emptyset, \text { if }\left|x_{2}\right|>\gamma .
\end{array}\right.
$$

Given $(t, x) \in \operatorname{dom}(V)$, for any $M$ large enough and for all $(\theta, \xi) \in \partial^{P} V(t, x)$ one has

$$
\begin{aligned}
& \theta+\min _{v \in\left(f(t, x)-A_{t, x}(x)\right) \cap M \mathbb{B}}\langle\xi, v\rangle \\
\leq & x_{1}\left(-\alpha x_{1}+\beta x_{2}\right)+x_{2}\left(-\beta x_{1}+x_{2}\right)-\gamma\left|x_{2}\right| \\
= & -\alpha x_{1}^{2}+\left|x_{2}\right|\left(\left|x_{2}\right|-\gamma\right) \\
\leq & 0 .
\end{aligned}
$$

Applying Theorem 4.1. we conclude that $V$ is a Lyapunov function for (53). 


\section{Applications for sweeping processes and Lur'e dynamical sys- tems}

In this section, we show that the obtained results in Sections 3 and 4 can be used to study two well-known problems: sweeping processes and Lur'e dynamical systems.

\subsection{State-dependent sweeping processes}

Let us consider the case $A_{t, x}=N_{C(t, x)}$, the normal cone of a moving set, where $C:[0, T] \times \mathbb{R}^{n} \rightrightarrows \mathbb{R}^{n}$ has non-empty closed convex values. Thanks to Lemma 2.2. Assumptions 1.1 is equivalent to

$$
d_{H}(C(t, x), C(s, y)) \leq L_{1}|t-s|+L_{2}\|x-y\|, \quad \forall t, s \in[0, T], x, y \in \mathbb{R}^{n},
$$

for some constant $L_{1} \geq 0,0 \leq L_{2}<1$. Our problem (1) becomes the classical state-dependent sweeping processes. Then Theorem 3.1 allows us to obtain the existence result which is accordant with [16]. The case of infinite Hilbert spaces can be done similarly with some compactness assumption. In [16], the authors provided some examples to show that the existence result can be lost if $L_{2} \geq 1$. So our upper bound for $L_{2}$ in problem (1) is optimal. In general, we do not have the uniqueness of solutions for state-dependent sweeping processes (see, e.g.,[16]). Here we give a uniqueness result under the hypo-monotonicity of the normal cone, which is a corollary of Theorem 3.2 .

Theorem 5.1 Let all the assumptions of Theorem 3.2 hold. Then for each $x_{0} \in C\left(t_{0}, x_{0}\right)$ with $0 \leq t_{0} \leq T$, the following differential inclusion

$$
\left\{\begin{array}{l}
\dot{x}(t) \in f(t, x(t))-N_{C(t, x(t))}(x(t)), \text { a.e. } t \in\left[t_{0}, T\right], \\
x\left(t_{0}\right)=x_{0},
\end{array}\right.
$$

has a unique solution on $\left[t_{0}, T\right]$.

Although the literature for the well-posedness of sweeping processes is immense, there is still no work studying Lyapunov stability for the state-dependent case. It is why the following corollary of Theorem 4.1 is interesting. Let us first introduce the admissible set for problem (56):

$$
\mathcal{A}_{2}:=\left\{\left(t_{0}, x_{0}\right) \in \mathbb{R}_{+} \times \mathbb{R}^{n}: x_{0} \in C\left(t_{0}, x_{0}\right)\right\} .
$$

Theorem 5.2 Let $V \in \Gamma_{w}\left([0, T] \times \mathbb{R}^{n}\right), W \in \Gamma_{+}\left([0, T] \times \mathbb{R}^{n}\right), a \geq 0$ and $\operatorname{dom}(V) \subset \mathcal{A}_{2}$. Then the following assertions are equivalent:

(i) For each $\left(t_{0}, x_{0}\right) \in \operatorname{dom}(V)$, we have

$$
e^{a\left(t-t_{0}\right)} V(t, x(t))+\int_{t_{0}}^{t} W(\tau, x(\tau)) d \tau \leq V\left(t_{0}, x_{0}\right) \quad \forall t \geq 0,
$$

where $x(\cdot):=x\left(\cdot ; t_{0}, x_{0}\right)$. 
(ii) For each $(t, x) \in \operatorname{dom}(V)$ and $(\theta, \xi) \in \partial^{P} V(t, x)$ we have

$$
\theta+\min _{v \in\left(f(t, x)-N_{C(t, x)}(x)\right) \cap M(x) \mathbb{B}}\langle\xi, v\rangle+a V(t, x)+W(t, x) \leq 0 .
$$

(iii) For each $(t, x) \in \operatorname{dom}(V)$ we have

$$
\left\{\begin{array}{l}
\sup _{(\theta, \xi) \in \partial^{P} V(t, x)}\left\{\theta+\min _{v \in\left(f(t, x)-N_{C(t, x)}(x)\right) \cap M(x) \mathbb{B}}\langle\xi, v\rangle+a V(t, x)+W(t, x)\right\} \leq 0, \\
\sup _{(\theta, \xi) \in \partial^{\infty} V(t, x)}\left\{\theta+\min _{v \in\left(f(t, x)-N_{C(t, x)}(x)\right) \cap M(x) \mathbb{B}}\langle\xi, v\rangle\right\} \leq 0 .
\end{array}\right.
$$

(iv) For each $(t, x) \in \operatorname{dom}(V)$, for any $M>0$ large enough, we have

$$
\left\{\begin{array}{c}
\sup _{(\theta, \xi) \in \partial^{P} V(t, x)}\left\{\theta+\min _{v \in\left(f(t, x)-N_{C(t, x)}(x)\right) \cap M \mathbb{B}}\langle\xi, v\rangle+a V(t, x)+W(t, x)\right\} \leq 0, \\
\sup _{(\theta, \xi) \in \partial^{\infty} V(t, x)}\left\{\theta+\min _{v \in\left(f(t, x)-N_{C(t, x)}(x)\right) \cap M \mathbb{B}}\langle\xi, v\rangle\right\} \leq 0 .
\end{array}\right.
$$

where $M(x)$ is defined in (37).

\subsection{State-dependent Lur'e dynamical systems}

Now we consider the class of state-dependent Lur'e dynamical systems (3) in the Introduction. It is known from (4) that we can reduce (3) into the following first order differential inclusions

$$
\left\{\begin{array}{l}
\dot{x}(t) \in g(t, x(t))-B \Phi(t, x(t), x(t)), \text { a.e. } t \in[0, T], \\
x\left(t_{0}\right)=x_{0},
\end{array}\right.
$$

where

$$
\Phi(t, x, y):=\left(F_{t, y}^{-1}+D\right)^{-1} C x, \quad(t, x, y) \in[0, T] \times \mathbb{R}^{2 n} .
$$

The admissible set for problem (61) is defined by

$$
\mathcal{A}_{3}:=\left\{\left(t_{0}, x_{0}\right) \in \mathbb{R}_{+} \times \mathbb{R}^{n}:\left(F_{t_{0}, x_{0}}^{-1}+D\right)^{-1} C x_{0} \neq \emptyset\right\} .
$$

Let us propose the following assumptions.

Assumption 3 For every $t \in[0, T]$ and $x \in \mathbb{R}^{n}$, the operator $F_{t, x}: \mathbb{R}^{n} \rightrightarrows \mathbb{R}^{n}$ be a maximal monotone operator and there exists $L_{F 1} \geq 0,0 \leq L_{F 2} \leq \frac{c_{2}}{\|C\|}$ such that

$$
\operatorname{dis}\left(F_{t, x}, F_{s, y}\right) \leq L_{F 1}|t-s|+L_{F 2}\|x-y\|, \quad \forall t, s \in[0, T],
$$

where $c_{2}$ is the smallest positive eigenvalue of $C^{T} C$.

Assumption 4 The matrix $D$ is positive semidefinite, $C^{T} C$ is full-rank and

$$
\operatorname{ker}\left(D+D^{T}\right) \subset \operatorname{ker}\left(P B-C^{T}\right)
$$

for some symmetric positive definite matrix $P$. 
Assumption 5 For all $t \geq 0$, if $\left(F_{t, y}^{-1}+D\right)^{-1} C x \neq \emptyset$ for some $x, y \in \mathbb{R}^{n}$, it holds that $\operatorname{rge}(D+$ $\left.D^{T}\right) \cap\left(F_{t, y}^{-1}+D\right)^{-1} C x \neq \emptyset$.

Assumption 6 For all $t \in[0, T], x \in \mathbb{R}^{n}: \operatorname{rge}(C) \cap \operatorname{rint}\left(\operatorname{rge}\left(F_{t, x}^{-1}+D\right)\right) \neq \emptyset$.

Assumption 7 The single-valued function $g:[0, T] \times \mathbb{R}^{n} \rightarrow \mathbb{R}^{n}$ is continuous and there exists $c_{f}>0$ such that

$$
\|g(t, x)\| \leq c_{f}(1+\|x\|), \quad \forall(t, x) \in[0, T] \times \mathbb{R}^{n} .
$$

The following lemmas are useful.

Lemma 5.1 Suppose that $C C^{T}$ is full-rank. Then for each $y \in \mathbb{R}^{m}$, we have

$$
\left\|C^{T} y\right\| \geq \frac{c_{2}}{\|C\|}\|y\| .
$$

where $c_{2}$ is the smallest positive eigenvalue of $C^{T} C$.

Proof. For all $y \in \mathbb{R}^{m}$, we have

$$
c_{2}\|y\|^{2} \leq\left\langle C C^{T} y, y\right\rangle \leq\|C\|\left\|C^{T} y\right\|\|y\|
$$

and the conclusion follows.

The following result is similar to [19, Lemma 11], where the case $F_{t, x} \equiv N_{C(t, x)}$ is considered. For the completeness, we recall it here.

Lemma 5.2 Suppose that Assumptions 3,4,5 are satisfied. Then we can find $\beta_{1}, \beta_{2}>0$ such that the single-valued minimal-norm function $\Phi^{0}:[0, T] \times \mathbb{R}^{2 n} \rightarrow \operatorname{rge}\left(D+D^{T}\right),(t, x, y) \mapsto \Phi^{0}(t, x, y)$ satisfies the following properties:

a) $\left\|\Phi^{0}(t, x, y)\right\| \leq \beta_{1}(1+\|x\|+\|y\|), \quad \forall(t, x, y) \in \operatorname{dom}\left(\Phi^{0}\right)$.

b) $\left\|\Phi^{0}\left(t_{1}, x_{1}, y_{1}\right)-\Phi^{0}\left(t_{2}, x_{2}, y_{2}\right)\right\|^{2} \leq \beta_{2}\left\|x_{1}-x_{2}\right\|^{2}+\beta_{2}\left(1+\left\|\Phi^{0}\left(t_{1}, x_{1}, y_{1}\right)\right\|+\left\|\Phi^{0}\left(t_{2}, x_{2}, y_{2}\right)\right\|\right)\left(\mid t_{1}-\right.$ $\left.t_{2} \mid+\left\|y_{1}-y_{2}\right\|\right), \quad \forall\left(t_{i}, x_{i}, y_{i}\right) \in \operatorname{dom}\left(\Phi^{0}\right), i=1,2$. In particular, the function $(t, x) \rightarrow \Phi^{0}(t, x, x)$ is continuous.

Proof. a) Let be given $(t, x, y) \in \operatorname{dom}\left(\Phi^{0}\right)$, which deduces that $\left(F_{t, y}^{-1}+D\right)^{-1} C x \neq \emptyset$. Using Assumption 5, we can find some $z_{0} \in \operatorname{rge}\left(D+D^{T}\right) \cap\left(F_{t, y}^{-1}+D\right)^{-1}(C x)=\operatorname{rge}\left(D+D^{T}\right) \cap \Phi(t, x, y)$. Let us prove that $\Phi^{0}(t, x, y)=z_{0} \in \operatorname{rge}\left(D+D^{T}\right)$. It is enough to show that $\left\|z_{1}\right\| \geq\left\|z_{0}\right\|$ for all $z_{1} \in \Phi(t, x, y)$. Note that we can always write uniquely $z_{1}=z_{1}^{i m}+z_{1}^{k e r}$ where $z_{1}^{i m} \in \operatorname{rge}(D+$ $\left.D^{T}\right), z_{1}^{k e r} \in \operatorname{ker}\left(D+D^{T}\right)$ satisfy $\left\langle z_{1}^{i m}, z_{1}^{k e r}\right\rangle=0$. We have

$$
z_{i} \in\left(F_{t, y}^{-1}+D\right)^{-1}(C x) \Leftrightarrow z_{i} \in F_{t, y}\left(C x-D z_{i}\right), i=0,1 .
$$

Using the monotonicity of $F_{t, y}$ and $D$, we imply that $\left\langle D\left(z_{0}-z_{1}\right), z_{0}-z_{1}\right\rangle=0$. It means that $z_{1}-z_{0}=z_{1}^{i m}+z_{1}^{k e r}-z_{0} \in \operatorname{ker}\left(D+D^{T}\right)$ and hence $z_{1}^{i m}-z_{0} \in \operatorname{ker}\left(D+D^{T}\right) \cap \operatorname{rge}\left(D+D^{T}\right)=\{0\}$. Therefore

$$
\left\|z_{1}\right\|^{2}=\left\|z_{1}^{i m}\right\|^{2}+\left\|z_{1}^{k e r}\right\|^{2}=\left\|z_{0}\right\|^{2}+\left\|z_{1}^{k e r}\right\|^{2} \geq\left\|z_{0}\right\|^{2},
$$

and one obtains that $\Phi^{0}(t, x, y)=z_{0} \in \operatorname{rge}\left(D+D^{T}\right)$. 
Fix $\left(t_{0}, x_{0}, x_{0}\right) \in \operatorname{dom}\left(\Phi^{0}\right)$, where $\left(t_{0}, x_{0}\right)$ is a point in $\mathcal{A}_{3}$. Using the definition of $\operatorname{dis}\left(F_{t, y}, F_{t_{0}, x_{0}}\right)$ and the fact that

$$
\begin{gathered}
\Phi^{0}\left(t_{0}, x_{0}, x_{0}\right) \in\left(F_{t_{0}, x_{0}}^{-1}+D\right)^{-1}\left(C x_{0}\right) \Leftrightarrow \Phi^{0}\left(t_{0}, x_{0}, x_{0}\right) \in F_{t_{0}, x_{0}}\left(C x_{0}-D \Phi^{0}\left(t_{0}, x_{0}, x_{0}\right)\right) \\
\Phi^{0}(t, x, y) \in\left(F_{t, y}^{-1}+D\right)^{-1}(C x) \Leftrightarrow \Phi^{0}(t, x, y) \in F_{t, y}\left(C x-D \Phi^{0}(t, x, y)\right)
\end{gathered}
$$

one has

$$
\begin{aligned}
& \left\langle C\left(x-x_{0}\right), \Phi^{0}(t, x, y)-\Phi^{0}\left(t_{0}, x_{0}, x_{0}\right)\right\rangle \\
\geq & \left\langle D\left(\Phi^{0}(t, x, y)-\Phi^{0}\left(t_{0}, x_{0}, x_{0}\right)\right), \Phi^{0}(t, x, y)-\Phi^{0}\left(t_{0}, x_{0}, x_{0}\right)\right\rangle \\
- & \left(1+\left\|\Phi^{0}(t, x, y)\right\|+\left\|\Phi^{0}\left(t_{0}, x_{0}, x_{0}\right)\right\|\right)\left(L_{1}\left|t-t_{0}\right|+L_{2}\left\|y-x_{0}\right\|\right) \\
\geq & c_{1}\left\|\Phi^{0}(t, x, y)-\Phi^{0}\left(t_{0}, x_{0}, x_{0}\right)\right\|^{2} \\
- & \left(1+\left\|\Phi^{0}(t, x, y)\right\|+\left\|\Phi^{0}\left(t_{0}, x_{0}, x_{0}\right)\right\|\right)\left(L_{1} T+L_{2}\left\|y-x_{0}\right\|\right),
\end{aligned}
$$

where $c_{1}>0$ is defined in Lemma 2.1. Hence there exists some $\beta>0$ such that

$$
\begin{aligned}
\left\|\Phi^{0}(t, x, y)\right\|^{2} & \leq\left\|\Phi^{0}(t, x, y)\right\|(\beta\|x\|+\beta\|y\|+\beta)+\beta(\|x\|+\beta\|y\|+1) \\
& \leq \beta\left(\left\|\Phi^{0}(t, x, y)\right\|+1\right)(\|x\|+\|y\|+1)
\end{aligned}
$$

and one obtains the conclusion with $\beta_{1}:=2 \beta+1$.

b) Similarly as in (67), we have for every $\left(t_{i}, x_{i}, y_{i}\right) \in \operatorname{dom}\left(\Phi^{0}\right), i=1,2$ that

$$
\begin{aligned}
& \left\langle C\left(x_{1}-x_{2}\right), \Phi^{0}\left(t_{1}, x_{1}, y_{1}\right)-\Phi^{0}\left(t_{2}, x_{2}, y_{2}\right)\right\rangle \geq c_{1}\left\|\Phi^{0}\left(t_{1}, x_{1}, y_{1}\right)-\Phi^{0}\left(t_{2}, x_{2}, y_{2}\right)\right\|^{2} \\
-\quad & \left(1+\left\|\Phi^{0}\left(t_{1}, x_{1}, y_{1}\right)\right\|+\left\|\Phi^{0}\left(t_{2}, x_{2}, y_{2}\right)\right\|\right)\left(L_{1}\left|t_{1}-t_{2}\right|+L_{2}\left\|y_{1}-y_{2}\right\|\right) .
\end{aligned}
$$

On the other hand

$$
\begin{aligned}
& \left\langle C\left(x_{1}-x_{2}\right), \Phi^{0}\left(t_{1}, x_{1}, y_{1}\right)-\Phi^{0}\left(t_{2}, x_{2}, y_{2}\right)\right\rangle \\
\leq & \frac{c_{1}}{2}\left\|\Phi^{0}\left(t_{1}, x_{1}, y_{1}\right)-\Phi^{0}\left(t_{2}, x_{2}, y_{2}\right)\right\|^{2}+\frac{\|C\|^{2}}{2 c_{1}}\left\|x_{1}-x_{2}\right\|^{2},
\end{aligned}
$$

and thus the conclusion follows.

Now we are ready for the well-posedness of (3).

Theorem 5.3 (Existence) Let Assumptions 3-7 hold. Then for each $\left(t_{0}, x_{0}\right) \in \mathcal{A}_{3}$, problem (3) has a solution.

Proof. The inclusion in (61) can be rewritten as

$$
\dot{x}(t) \in g(t, x(t))-\left(B-C^{T}\right) \Phi(t, x(t), x(t))-C^{T} \Phi\left(t, x(t), x(t)=f(t, x(t))-A_{t, x(t)}(x(t))\right.
$$

where

$$
f(t, x):=g(t, x(t))-\left(B-C^{T}\right) \Phi(t, x(t), x(t))
$$

and

$$
A_{t, y}(x):=C^{T} \Phi(t, x, y)=C^{T}\left(F_{t, y}^{-1}+D\right)^{-1} C x .
$$


Then $A$ and $f$ satisfy Assumptions 1.2 and 2 respectively (see Lemma 5.2). It remains to check that $A$ satisfies Assumption 1.1. Indeed, we have

$$
\begin{aligned}
& \operatorname{dis}\left(A_{t_{1}, y_{1}}, A_{t_{2}, y_{2}}\right) \\
= & \sup \left\{\frac{\left\langle C^{T} x_{1}^{*}-C^{T} x_{2}^{*}, x_{2}-x_{1}\right\rangle}{1+\left\|C^{T} x_{1}^{*}\right\|+\left\|C^{T} x_{2}^{*}\right\|}: x_{i}^{*} \in\left(F_{t_{i}, y_{i}}^{-1}+D\right)^{-1} C x_{i}\right\} \\
= & \sup \left\{\frac{\left\langle x_{1}^{*}-x_{2}^{*}, C x_{2}-C x_{1}\right\rangle}{1+\left\|C^{T} x_{1}^{*}\right\|+\left\|C^{T} x_{2}^{*}\right\|}: x_{i}^{*} \in F_{t_{i}, y_{i}}\left(C x_{i}-D x_{i}^{*}\right)\right\} \\
\leq & \sup \left\{\frac{\left\langle x_{1}^{*}-x_{2}^{*},\left(C x_{2}-D x_{2}^{*}\right)-\left(C x_{1}-D x_{1}^{*}\right)\right\rangle}{1+\left\|x_{1}^{*}\right\|+\left\|x_{2}^{*}\right\|} \frac{1+\left\|x_{1}^{*}\right\|+\left\|x_{2}^{*}\right\|}{1+\left\|C^{T} x_{1}^{*}\right\|+\left\|C^{T} x_{2}^{*}\right\|}: x_{i}^{*} \in F_{t_{i}, y_{i}}\left(C x_{i}-D x_{i}^{*}\right)\right\} \\
& (\operatorname{since} D \text { is positive semidefinite) } \\
\leq & \frac{\|C\|}{c_{2}} \sup \left\{\frac{\left\langle x_{1}^{*}-x_{2}^{*},\left(C x_{2}-D x_{2}^{*}\right)-\left(C x_{1}-D x_{1}^{*}\right)\right\rangle}{1+\left\|x_{1}^{*}\right\|+\left\|x_{2}^{*}\right\|}: x_{i}^{*} \in F_{t_{i}, y_{i}}\left(C x_{i}-D x_{i}^{*}\right)\right\} \\
& (\text { using Lemma } 5.1) \\
\leq & \frac{\|C\|}{c_{2}} \operatorname{dis}\left(F_{t_{1}, y_{1}}, F_{t_{2}, y_{2}}\right)\left(\text { using the definition of dis }\left(F_{t_{1}, y_{1}}, F_{t_{2}, y_{2}}\right)\right) \\
\leq & \frac{\|C\|}{c_{2}}\left(L_{1}\left|t_{1}-t_{2}\right|+L_{2}\left\|y_{1}-y_{2}\right\|\right) \\
\leq & \left(L_{1}^{\prime}\left|t_{1}-t_{2}\right|+L_{2}^{\prime}\left\|y_{1}-y_{2}\right\|\right),
\end{aligned}
$$

where $L_{1}^{\prime}:=\frac{\|C\| L_{1}}{c_{2}}$ and $L_{2}^{\prime}:=\frac{\|C\| L_{2}}{c_{2}}<1$. Thus, the proof is completed.

Remark 5.1 (i) If $F_{t, x} \equiv N_{C(t, x)}$, the full-rankness of $C C^{T}$ can be relaxed by the condition $\operatorname{rge}(D) \subset \operatorname{rge}(C)$ by using nice property of the normal cone [19].

(ii) The state-dependent sweeping process is also a special case of (3) when $F_{t, x} \equiv N_{C(t, x)}$ and $B=C=I, D=0$.

Theorem 5.4 (Uniqueness) Let all assumptions of Theorem 5.3 hold. In addition, suppose that $F_{t, x}(\cdot)=G_{t}\left(\cdot+g_{1}(t, x)\right)$ where $G_{t}: \mathbb{R}^{n} \rightrightarrows \mathbb{R}^{n}$ is a maximal monotone operator for each $t \in[0, T]$ and $g_{1}:[0, T] \times \mathbb{R}^{n} \rightarrow \operatorname{rge}\left(D+D^{T}\right)$ and $g$ are Lipschitz continuous in bounded sets w.r.t the second variable. Then for each $\left(t_{0}, x_{0}\right) \in \mathcal{A}_{3}$, problem (3) has a unique solution.

Proof. Using Theorem 3.2 , it is sufficient to show that $A$ defined in $(69)$ is hypo-monotone in bounded sets. Indeed, given $M>0$, for all $t \in[0, T], x_{i} \in \mathbb{R}^{n} \cap M \mathbb{B}, x_{i}^{*} \in A_{t, x_{i}}\left(x_{i}\right), i=1,2$ we have $y_{i}=C^{T} z_{i}^{*}$ where

$$
z_{i}^{*} \in\left(F_{t, x_{i}}^{-1}+D\right)^{-1} C x_{i} \Leftrightarrow z_{i}^{*} \in F_{t, x_{i}}\left(C x_{i}-D z_{i}^{*}\right)=G_{t}\left(C x_{i}-D z_{i}^{*}+g_{1}(t, x)\right) .
$$

Thank to the monotonicity of $G_{t}$, one has

$$
\left\langle z_{1}^{*}-z_{2}^{*},\left(C x_{1}-D z_{1}^{*}+g_{1}\left(t, x_{1}\right)-\left(C x_{2}-D z_{2}^{*}+g_{1}\left(t, x_{2}\right)\right\rangle \geq 0 .\right.\right.
$$

Therefore,

$$
\begin{aligned}
\left\langle x_{1}^{*}-x_{2}^{*}, x_{1}-x_{2}\right\rangle & =\left\langle C^{T} z_{1}^{*}-C^{T} z_{2}^{*}, x_{1}-x_{2}\right\rangle \\
& \geq\left\langle D\left(z_{1}^{*}-z_{2}^{*}\right), z_{1}^{*}-z_{2}^{*}\right\rangle-\left\langle z_{1}^{*}-z_{2}^{*}, g_{1}\left(t, x_{1}\right)-g_{1}\left(t, x_{2}\right)\right\rangle \\
& \geq c_{1}\left\|z_{1}^{* i m}-z_{2}^{* i m}\right\|^{2}-l_{M}\left\|z_{1}^{* i m}-z_{2}^{* i m}\right\|\left\|x_{1}-x_{2}\right\| \\
& \geq-\frac{l_{M}^{2}}{4 c_{1}}\left\|x_{1}-x_{2}\right\|^{2}
\end{aligned}
$$


where $c_{1}>0$ is defined in Lemma 2.1, $l_{M}$ is the Lipschitz constant of $g_{1}$ on the ball $M \mathbb{B}$ and $z^{* i m}$ denotes the projection of $z^{*}$ onto $\operatorname{rge}\left(D+D^{T}\right)$. The proof is therefore completed.

We also have a characterization for nonsmooth Lyapunov pairs associated with problem (3), which is a consequence of Theorem 4.1 .

Theorem 5.5 Let $V \in \Gamma_{w}\left([0,+\infty) \times \mathbb{R}^{n}\right), W \in \Gamma_{+}\left([0,+\infty) \times \mathbb{R}^{n}\right), a \geq 0$ and $\operatorname{dom}(V) \subset \mathcal{A}_{3}$. Then the following assertions are equivalent:

(i) For each $\left(t_{0}, x_{0}\right) \in \operatorname{dom}(V)$, we have

$$
e^{a\left(t-t_{0}\right)} V(t, x(t))+\int_{t_{0}}^{t} W(\tau, x(\tau)) d \tau \leq V(t, x) \quad \forall t \geq 0,
$$

where $x(\cdot):=x\left(\cdot ; t_{0}, x_{0}\right)$.

(ii) For each $(t, x) \in \operatorname{dom}(V)$ and $(\theta, \xi) \in \partial^{P} V(t, x)$ we have

$$
\theta+\underset{v \in\left(g(t, x)-B\left(F_{t, x}^{-1}+D\right)^{-1} C x\right) \cap M(x) \mathbb{B}}{\min }\langle\xi, v\rangle+a V(t, x)+W(t, x) \leq 0 .
$$

(iii) For each $(t, x) \in \operatorname{dom}(V)$ we have

$$
\left\{\begin{array}{l}
\sup _{(\theta, \xi) \in \partial^{P} V(t, x)}\left\{\theta+\min _{v \in\left(g(t, x)-B\left(F_{t, x}^{-1}+D\right)^{-1} C x\right) \cap M(x) \mathbb{B}}\langle\xi, v\rangle+a V(t, x)+W(t, x)\right\} \leq 0, \\
\sup _{(\theta, \xi) \in \partial^{\infty} V(t, x)}\left\{\theta+\min _{v \in\left(g(t, x)-B\left(F_{t, x}^{-1}+D\right)^{-1} C x\right) \cap M(x) \mathbb{B}}\langle\xi, v\rangle\right\} \leq 0 .
\end{array}\right.
$$

(iv) For each $(t, x) \in \operatorname{dom}(V)$, for any $M>0$ large enough, we have

$$
\left\{\begin{array}{c}
\sup _{(\theta, \xi) \in \partial^{P} V(t, x)}\left\{\theta+\min _{v \in\left(g(t, x)-B\left(F_{t, x}^{-1}+D\right)^{-1} C x\right) \cap M \mathbb{B}}\langle\xi, v\rangle+a V(t, x)+W(t, x)\right\} \leq 0, \\
\sup _{(\theta, \xi) \in \partial^{\infty} V(t, x)}\left\{\theta+\min _{v \in\left(g(t, x)-B\left(F_{t, x}^{-1}+D\right)^{-1} C x\right) \cap M \mathbb{B}}\langle\xi, v\rangle\right\} \leq 0 .
\end{array}\right.
$$

where $M(x)$ is defined in (37).

Proof. Let us note that for each $(t, x) \in[0,+\infty) \times \mathbb{R}^{n}$, we have

$$
f(t, x)-A_{t, x}(x)=g(t, x)-B\left(F_{t, x}^{-1}+D\right)^{-1} C x .
$$

Therefore, the conclusion follows by using Theorem 4.1 .

\section{Conclusion}

In the paper, we introduce and study the well-posedness as well as nonsmooth Lyapunov pairs for a class of state-dependent maximal monotone differential inclusions with some illustrative examples. The obtained results can be used to deal with the Lyapunov stability for state-dependent sweeping processes and Lur'e dynamical systems for the first time. Well-posedness of state-dependent Lur'e systems involving general maximal monotone operators is also considered here. 


\section{References}

[1] S. Adly, A. Hantoute, B. K. Le, Nonsmooth Lur'e Dynamical Systems in Hilbert Spaces, Set-Valued Var. Anal, vol 24, iss. 1, 13-35, 2016.

[2] S. Adly, A. Hantoute, M. Thera, Nonsmooth Lyapunov pairs for infinite-dimensional first-order differential inclusions, Nonlinear Anal. 75 (2012), 985-1008.

[3] S. Adly, T. Haddad, L. Thibault, Convex Sweeping Process in the framework of Measure Differential Inclusions and Evolution Variational Inequalities, Math. Program. Ser. B 148 (2014), 5-47.

[4] S. AdLy, B. K. LE, Non-convex sweeping processes involving maximal monotone operators, Optimizations, vol. 66 (9), 1465-1486, 2017.

[5] J. P. Aubin, A. Cellina, Differential Inclusions. Set-Valued Maps and Viability Theory, Spinger-Verlag, Berlin, 1984.

[6] H. Brezis, Opérateurs Maximaux Monotones et Semi-groupes de Contractions dans les Espaces de Hilbert, Math. Studies 5, North-Holland American Elsevier (1973).

[7] B. Brogliato Absolute stability and the Lagrange-Dirichlet theorem with monotone multivalued mappings, Systems and Control Letters 2004, 51 (5), 343-353.

[8] B. Brogliato, D. Goeleven, Well-posedness, stability and invariance results for a class of multivalued Lur'e dynamical systems, Nonlinear Analysis: Theory, Methods and Applications, vol. 74, pp. 195-212, 2011.

[9] B. Brogliato, D. Goeleven, Existence, uniqueness of solutions and stability of nonsmooth multivalued Lur'e dynamical systems, Journal of Convex Analysis, vol. 20, no. 3, pp. 881-900, 2013.

[10] M. K. Camlibel, J. M. Schumacher, Linear passive systems and maximal monotone mappings, Math. Program. 157 (2), pp 397-420, 2016.

[11] F. H. Clarke, Y. S. Ledyaev, R. J. Stern, P. R. Wolenski, Nonsmooth Analysis and Control Theory, vol. 178 of Graduate Texts in Mathematics. Springer, New York, 1998.

[12] A. Hantoute, E. Vilches Lyapunov pairs for perturbed sweeping processes, to appear in Optimization Letters.

[13] N. Kenmochi, Solvability of nonlinear evolution equations with time-dependent constraints and applications, Bull. Fac. Educ. Chibo Univ. 30, 1-81 (1981).

[14] A. Krasnosel'skimi, V. Pokrovskima, Systems with Hysteresis. Springer, Berlin (1988).

[15] M. Kunze, M.D.P. Monteiro Marques, BV solutions to evolution problems with timedependent domains, Set-Valued Anal., 5 (1997), pp. 57-72.

[16] M. Kunze, M.D.P. Monteiro Marques, An introduction to Moreau's sweeping process , in "Impacts in Mechanical Systems. Analysis and Modelling", (B. Brogliato, Ed), 1-60, Springer, Berlin, 2000. 
[17] D. Azzam-Laouir, C. Castaing, M.D.P Monteiro Marques, Perturbed Evolution Problems with Continuous Bounded Variation in Time and Applications, Set-Valued Var. Anal (2018) 26, 693-728.

[18] B. K. Le, On Properties of Differential Inclusions with Prox-regular Sets, Pac. J. Optim. 13 (2017), 17-27.

[19] B. K. LE , Lur'e dynamical systems with state-dependent set-valued feedback, submitted.

[20] M. R. Liberzon, Essays on the absolute stability theory, Automation and Remote Control, vol. 67, no 10, pp. 1610-1644, October 2006.

[21] B. Mordukhovich, Variational Analysis and Generalized Differentiation I, Springer, 2006.

[22] J. J. Moreau, Proximité et dualité dans un espace hilbertien, Bull. Soc. Math. France, 93 (1965), 273-299.

[23] J. J. Moreau, Sur l'evolution d'un système élastoplastique, C. R. Acad. Sci. Paris Sér. A-B, 273 (1971), A118-A121.

[24] J. J. Moreau, Rafle par un convexe variable I, Sém. Anal. Convexe Montpellier (1971), Exposé 15.

[25] J. J. Moreau, Rafle par un convexe variable II, Sém. Anal. Convexe Montpellier (1972), Exposé 3.

[26] N. H. Pavel, Nonlinear evolution operators and semigroups, Lecture Notes in Mathematics, 1260. Springer, New York (1987).

[27] A. Tanwani, B. Brogliato, C. Prieur Stability and observer design for Lur'e systems with multivalued, non-monotone, time-varying nonlinearities and state jumps, SIAM J. Control Opti., Vol. 52, No. 6, pp. 3639-3672, 2014.

[28] A. Tanwani, B. Brogliato, C. Prieur Well-Posedness and Output Regulation for Implicit Time-Varying Evolution Variational Inequalities, SIAM J. Control Opti., Vol. 56, No. 2, pp. 751-781, 2018.

[29] H. TANAKA, Stochastic differential equations with reflecting boundary conditions in convex regions, Hiroshima math. J. 9, 163-177 (1979).

[30] L. Thibault, Sweeping process with regular and nonregular sets, J. Differ. Equ. 193 (2003), $1-23$.

[31] L. Thibault, Regularization of nonconvex sweeping process in Hilbert space, Set-valued Anal. 16 (2008), 319-333.

[32] A. A. Vladimirov, Nonstationary dissipative evolution equations in a Hilbert space, Nonlinear Anal. 17 (1991) 499-518. 